\title{
Diabetic ketoacidosis
}

Ketan K. Dhatariya ${ }^{1,2}$, Nicole S. Glaser ${ }^{3}$, Ethel Codner ${ }^{4}$ and Guillermo E. Umpierrez ${ }^{5 \dagger}$

${ }^{1}$ Elsie Bertram Diabetes Centre, Norfolk and Norwich University Hospitals NHS Foundation Trust, Colney Lane, Norwich, Norfolk, UK

${ }^{2}$ Norwich Medical School, University of East Anglia, Norfolk, UK

${ }^{3}$ Department of Pediatrics, University of California Davis, School of Medicine, Sacramento, CA, USA

${ }^{4}$ Institute of Maternal and Child Research, School of Medicine, University of Chile, Santiago, Chile

${ }^{5}$ Diabetes \& Endocrinology, Emory University School of Medicine, Atlanta, GA, USA

†email: geumpie@emory.edu

\section{Author contributions}

Introduction (G.E.U., N.S.G., K.K.D., E.C.); Epidemiology (G.E.U., N.S.G., K.K.D., E.C.); Mechanisms/pathophysiology (G.E.U, N.S.G, K.K.D, E.C); Diagnosis, screening and prevention (G.E.U., N.S.G., K.K.D., E.C.); Management (G.E.U., N.S.G., K.K.D., E.C.); Quality of life (N.S.G.); Outlook (N.S.G, E.C); Overview of Primer (G.E.U). All named authors meet the International Committee of Medical Journal Editors (ICMJE) criteria for authorship for this article, take responsibility for the integrity of the work as a whole, and have given their approval for this version to be published.

\section{Competing interests}

K.K.D. is an employee of the UK National Health Service.

N.S.G. has grants from American Diabetes Association (ADA, 1-17-IBS-186) and from the National Institute of Diabetes and Digestive and Kidney Diseases (NIDDK, U34DK123894).

E.C. is partly funded by Fondo Nacional de Ciencia y Tecnología (FONDECYT) grant \#1170895 from the Government of Chile.

G.E.U. is partly supported by research grants from the National Center for Advancing Translational Sciences of the National Institutes of Health $(\mathrm{NIH})$ under Award Number UL1TR002378 from the Clinical and Translational Science Award program and an NIH grant 
U30, P30DK11102, and has received research grant support to Emory University for investigator-initiated studies from Dexcom, Novo Nordisk and Sanofi.

\section{Publisher's note}

Springer Nature remains neutral with regard to jurisdictional claims in published maps and institutional affiliations.

\section{Abstract}

Diabetic ketoacidosis (DKA) is the most common acute hyperglycaemic emergency in people with diabetes mellitus. A diagnosis of DKA is confirmed when all of the three criteria are present -'D', either elevated blood glucose levels or a family history of diabetes mellitus; 'K', the presence of high urinary or blood ketoacids; and 'A', a high anion gap metabolic acidosis. Early diagnosis and management is paramount to improve patient outcome. The mainstays of treatment include restoration of circulating volume, insulin therapy, electrolyte replacement and treatment of any underlying precipitating event. Without optimal treatment, DKA remains a condition with an appreciable, although largely preventable morbidity and mortality. In this Primer, we discuss the epidemiology, pathogenesis, risk factors and diagnosis of DKA, as well as we provide practical recommendations for management of DKA in adults and children. 


\section{[H1] Introduction}

Diabetic ketoacidosis (DKA) is the most common acute hyperglycaemic emergency in people with diabetes mellitus. DKA is the consequence of an absolute (that is, total absence of) or relative (that is, levels insufficient to supress ketone production) lack of insulin and concomitant elevation of counter-regulatory hormones, usually resulting in the triad of hyperglycaemia, metabolic acidosis and ketosis (elevated levels of ketones in the blood or urine; serum ketone concentration of $>3.0 \mathrm{mmol} / \mathrm{l}$ ), often accompanied by varying degrees of circulatory volume depletion [G]. DKA occurs mostly in people with uncontrolled type 1 diabetes mellitus (T1DM, which results from the autoimmune destruction of the $\beta$-cells of the islets of Langerhans), but can also occur in adults with poorly controlled type 2 diabetes mellitus (T2DM, a result of impaired insulin secretion or action) under stressful conditions such as acute medical or surgical illnesses and, in adolescents, new onset T2DM (also known as ketosis-prone T2DM) (Figure 1). Although any illness or physiological stress can precipitate DKA, the most frequent causes are infections, particularly urinary tract infections and gastroenteritis ${ }^{1,2}$.

DKA was previously considered to be a key clinical feature of T1DM, but has been documented in children and adults with newly diagnosed T2DM ${ }^{2,3}$. Although ketosis-prone T2DM can occur in all populations, epidemiological data suggest that people of African or Hispanic origin seem to be at greater risk $^{2}$. This predisposition likely has a genetic component, but this has yet to be elucidated. Most often individuals with ketosis-prone T2DM have obesity and a strong family history of T2DM and evidence of insulin resistance. Despite presenting with DKA and decreased insulin concentrations, on immunological testing these individuals have the same frequency of the typical autoimmune markers of T1DM such as islet cell, insulin, glutamic acid decarboxylase, and protein tyrosine phosphatase autoantibodies as those who present with HHS and their $\beta$-cell function recovers with restoration of insulin secretion quickly after treatment ${ }^{2}$. Thus, individuals with ketosis-prone T2DM can often go back to oral glucose-lowering medication, without the need for continuing insulin therapy. DKA is associated with significant morbidity and utilization of health care resources, accounting for $4-9 \%$ of all hospital discharges among those with a diagnosis of diabetes as the primary cause for their acute hospital admission ${ }^{4}$. DKA remains an expensive condition to treat. In the USA, a single episode of DKA is estimated to cost $\sim 26,566\left(\operatorname{Ref}^{5}\right)$. In the UK, the cost of one DKA episode is estimated to be $£ 2,064$ in adults and $£ 1,387$ in adolescents (11-18 years of age $)^{6,7}$. 
The criteria used to define DKA differ in different parts of the world (Table 1). In 2001, the American Diabetes Association (ADA) expanded the definition of DKA to include mild metabolic acidosis, hyperglycaemia and positive ketone tests ${ }^{8,9}$ (Table 1). Although all the definitions of DKA concur by saying that all three components need to be present, the glucose concentrations and method of documenting ketosis vary. Additionally, all guidelines agree that venous or arterial $\mathrm{pH}$ should be $<7.30$. Early diagnosis and treatment are paramount to improve patient outcomes. In developed countries, the risk of death resulting from DKA is $<1 \%$ in children and adults ${ }^{10,11}$ whereas in developing countries, mortality rates are much higher, with reported rates as high as $3-13 \%$ in children ${ }^{12}$. Among adults, DKA-related deaths occur primarily in older persons $(>60$ years of age) or in those with severe precipitating illnesses ${ }^{1}$. In children, the majority of DKArelated deaths result from cerebral injuries or cerebral oedema. Evidence-based treatment strategies include correction of fluid deficits, insulin therapy, potassium repletion and correction of the precipitating factor.

The other hyperglycaemic emergency that occurs is hyperosmolar hyperglycaemic state, which has a distinct pathophysiology to DKA (Box 1).

This Primer aims to provide up to date knowledge on the epidemiology, pathophysiology, clinical presentation, management of DKA. In addition, we also discuss prevention measures after discharge in adults and children with DKA.

\section{[H1] Epidemiology}

As the majority of people with DKA are hospitalized, most epidemiological data comes from hospital discharge coding. Among adults, two-thirds of episodes of DKA occur in people diagnosed with T1DM and one-third occur in those with T2DM ${ }^{3,11,13}$. In children $(<18$ years of age), DKA commonly occurs at the initial diagnosis of T1DM, with incidence varying in different populations from $13 \%$ to $80 \%{ }^{14-16}$. Adolescents with T2DM also present with DKA, although less frequently than children with $\mathrm{T}_{\mathrm{DDM}}{ }^{14}$. In addition, the frequency of DKA at diagnosis correlates inversely with the frequency of T1DM in the population, suggesting that the more frequent T1DM occurs in the general population, the more likely that symptoms of new onset are recognised before it becomes an episode of DKA ${ }^{17-19}$. DKA occurs as the earliest presentation of diabetes in children $<5$ years of age, and in people who do not have easy access to medical care for 
economic or social reasons ${ }^{20-22}$. Among individuals ( between 4.6 to 19.8 years of age), who were antibody negative and with median BMI z-score [G] $2.3(2.0,2.6), 11 \%$ presented with ketosisprone $\mathrm{T}_{2} \mathrm{DM}^{23}$. The percentage of adults with ketosis-prone T2DM is unknown; however, since the early 2000s, the prevalence of ketosis-prone T2DM worldwide has increased ${ }^{3,13}$. Studies investigating autoimmunity in ketosis-prone T2DM that have suggested an association between developing the condition and full-length tyrosine phosphatase IA-2 antibody (IA-2FL) or its extracellular domain (IA-2EC) ${ }^{24}$. Thus, individuals with genetic predisposition might be at greater risk of developing ketosis-prone T2DM.

Epidemiological studies in the USA and Europe revealed increasing hospitalizations for DKA in adults $^{10,13,25}$. In 2014, the US Centers for Disease Control and Prevention reported a total of 188,950 cases of $\mathrm{DKA}^{10}$. Between 2000 and 2009, an average decline of $1.1 \%$ in the annual ageadjusted DKA hospitalization rate was noted among people with any form of diabetes mellitus between ${ }^{10}$. However, the estimated average annual hospitalization rate increased to $6.3 \%$ between 2009 and 2014, that is, a rise of $54.9 \%$ in this period (from 19.5 to 30.2 per 1,000 persons). This increase was observed across all age groups and sexes. The highest hospitalization rates were in individuals $<45$ years of age, which might be attributed to poor control (44.3 per 1,000 persons in 2014) and lowest in persons >65 years of age for reasons unknown (<2.0 per 1,000 persons in 2014) ${ }^{10}$. The causes of increased DKA hospitalizations are not clear, but might relate to changes in DKA definition ${ }^{8,9}$, use of new medications associated with increased DKA risk and lower thresholds for hospitalization (that is, admission of individuals with less serious disease) $)^{10,13}$.

The rise in hospitalizations for DKA in the USA parallels the increased trend observed in the UK, Australia, New Zealand and Denmark ${ }^{11,26,27}$. A study from the UK examined nationally representative data in those with existing T1DM and T2DM using the Clinical Practice Research Datalink and the Hospital Episode Statistics databases between 1998 and 2013 (Ref ${ }^{11}$ ). The study found that the incidence of DKA was highest in adults between 18 and 24 years of age within 1 year of diagnosis, potentially suggesting a need for greater education on managing their diabetes at the time of diagnosis. In agreement with these reports, a systematic review ${ }^{25}$ reported worldwide incidence of $8-51.3$ cases per 1,000 patient-years in individuals with T1DM, which has shown to be the highest in men between 15 to 39 years of $a g e^{28}$. These data made no distinction between first or recurrent (an individual presenting with $>1$ episode at any time after their first event) episodes of DKA. Furthermore, the Guangdong Type 1 Diabetes Translational Study 
Group reported a much higher incidence across China (263 per 1,000 patient-years), which the investigators attributed to differences in national health care systems where people with T1DM have limited access to routine health care as well as infrequent self-monitoring of blood glucose $^{29}$. However, in jurisdictions such as Taiwan, Germany and Italy, DKA hospitalization rates have decreased ${ }^{30-32}$. The reasons for this decrease are unknown, but might be due to improvements in access to healthcare and/or increased recognition of the early signs of hyperglycaemia and DKA.

Recurrent DKA accounts for a substantial portion of the hospitalizations amongst adults with diabetes mellitus; $66 \%$ for T1DM and 35\% for T2DM in the UK ${ }^{11}$. However, a study in the USA reported recurrent DKA in $21.6 \%$ of adults with T1DM or T2DM between 18 and 89 years of age. Of those with recurrent DKA, $16 \%$ had been hospitalized at more than one hospital ${ }^{33}$, implying that patients do not get continuity of care and that their care is fragmented. Recurrent DKA often occurs in a small number of adults or children who have behavioural, social or psychological problems who make up a disproportionate number of DKA admissions ${ }^{33,34}$.

In developed countries, hospital case-fatality rates have declined over time with current reported mortality rates of $<1 \%$ were observed across all age groups and sexes ${ }^{10,35}$. However, DKA is the leading cause of mortality among children and adults $<58$ years old with T1DM, accounting for $>50 \%$ of all deaths in children with diabetes mellitus ${ }^{36}$. Mortality increases substantially in those with comorbidities and with ageing, reaching $8-10 \%$ in those $>65-75$ years of age $e^{1,37}$. The highest rates of DKA have been suggested to occur in regions least able to afford healthcare ${ }^{38}$. Mortality might also be higher in these populations, for example, data from India showed a $30 \%$ mortality in those presenting with $\mathrm{DKA}^{39}$ and studies from sub-Saharan Africa have reported similarly high mortality (26-41.3\%), whereas a study from Jamaica reported a mortality of $6.7 \%{ }^{39-}$ 41. Limited resources in the treating hospital, late presentation or higher case load in larger institutions might contribute to the higher mortality.

\section{[H2] Risk factors}

In adults with known diabetes mellitus, precipitating factors for DKA include infections, intercurrent illnesses such as acute coronary syndrome, insulin pump issues (for example, dislodgement or blockage of infusion sets), and poor adherence and noncompliance with insulin therapy (Table 2) ${ }^{1,35}$. Several new studies have emphasized the impact of poor treatment adherence on the incidence of DKA. For example, in the USA, among urban Afro-Caribbean 
populations and in underinsured people, noncompliance was the principal cause for the development of $\mathrm{DKA}^{42}$. As a result, poor adherence to insulin treatment accounted for $>50 \%$ of DKA admissions to a large urban hospital ${ }^{33,42}$. A study reported that persons without health insurance or with Medicaid alone (in the USA) had hozpitalisation rates 2-3 times higher for DKA than those with private insurance. A study examining two community hospitals in Chicago, IL, identified that most cases of DKA were caused by people with diabetes mellitus omitting their insulin (failure to administer insulin as directed) and medical illness accounted for less than onethird of admissions ${ }^{33}$. In the UK, the most frequent cause of DKA was infection, followed by noncompliance ${ }^{35}$. Other conditions that are known to precipitate DKA include myocardial infarction, cerebrovascular accidents, pancreatitis, alcohol misuse, pulmonary embolism and trauma ${ }^{1,8,35}$. The risk factors for recurrent DKA include low socioeconomic status, adolescence, female sex (possibly due to a higher incidence of deliberate insulin omission, psychological issues, eating disorders, and body dysmorphia ${ }^{43}$ ), high glycated haemoglobin (HbA1c), previous episodes of DKA and a history of mental health problems ${ }^{44-49}$.

In children, lack of prompt recognition of new-onset T1DM by healthcare providers increases the risk of DKA at diagnosis ${ }^{50}$. Among children with known T1DM, the majority of DKA episodes are caused by insulin omission with a minority of episodes occurring in association with infections most often gastrointestinal infections with vomiting and an inability to keep hydrated ${ }^{51}$. Risk factors for DKA in children with known diabetes mellitus include poor diabetes control, previous episodes of DKA, unstable or challenging family or social circumstances; adolescent age, being a peripubertal girl, and having limited access to medical services ${ }^{52,53}$. A study showed that in the USA and in India, a small proportion (5.5\% and 6.6\%, respectively) of people aged $\leq 19$ years who are eventually diagnosed with T2DM present with DKA ${ }^{54}$. Whether this is ketosis-prone T2DM is unknown as genetic analyses on these individuals is unavailable.

Psychological factors also influence the likelihood of developing DKA ${ }^{55,56}$. A report of $\sim 350$ adolescent girls and women (aged 13-60 years) suggested that disordered eating and was a contributing factor in $\sim 20 \%$ of recurrent episodes of $D K A^{57}$. Furthermore, $\sim 30 \%$ of young women (15 \pm 2 years of age) with T1DM have been suggested to have an eating disorder ${ }^{58}$. When questioned, the women omitted insulin because of a fear of weight gain with good glycaemic control, diabetes-related distress, fear of hypoglycaemia, and rebellion from authority ${ }^{59}$.

\section{[H3] Pharmacological risk factors.}


As mentioned, insulin mismanagement or omission can lead to DKA. Most often treatment involves insulin given in a multiple dose regimen. However, data from the UK National Paediatric Diabetes Audit shows that insulin pump use is also associated with an increased risk of DKA in the $<18$ year old population ${ }^{60}$. DKA has also been reported in people with diabetes mellitus treated with sodium-glucose transport protein 2 (SGLT2) inhibitors. Results from randomized controlled trials (RCTs) have indicated that DKA is rare in patients with T2DM treated with SGLT2 inhibitors (incidence of $0.16-0.76$ events per 1,000 patientyears ${ }^{61}$ ). Several RCTs, however, have reported a higher risk of SGLT2 inhibitor-associated ketosis in adults with T1DM (5-12\%) ${ }^{62-64}$ and an incidence of DKA in $~ 3-5 \%$ in those with T1DM treated with SGLT2 inhibitors ${ }^{62,65}$. The incidence of DKA in those receiving placebo in these RCTs of people with T1DM was $0-1.9 \%{ }^{64}$ and DKA occurred despite the use of measures designed to minimize the risk of ketosis. These risk mitigation strategies have been described elsewhere ${ }^{66,67}$. With the regulatory approval of SGLT2 inhibitors for use in patients with overweight and T1DM in Europe ${ }^{68}$, the actual rates of DKA outside of a clinical trial setting remain to be determined. The only other drug licensed in the USA for use in people with T1DM is pramlintide ${ }^{69}$. The use of this drug is not associated with the development of DKA, but is seldom used because it needs to be injected at each meal as a separate injection to insulin, causes nausea, and hypoglycaemia might occur if the insulin to carbohydrate ratio is incorrect. Thus, there is a need to develop better adjunctive treatments alongside insulin for people with T1DM.

Data from the T1DM exchange registry in the USA has shown that cannabis use is associated with an increased risk of developing $\mathrm{DKA}^{70}$. In addition, drugs that affect carbohydrate metabolism such as corticosteroids, sympathomimetic agents (used in nasal decongestants) and pentamidine (an antimicrobial agent most frequently used to treat protozoal infection or pneumonia) can precipitate the development of $\mathrm{DKA}^{1,9}$. Atypical antipsychotic agents have been associated with weight gain and T2DM, but are also associated with DKA, which occur acutely even in the absence of weight gain ${ }^{71,72}$. Cancer treatment using immune check-point inhibitors (ICls), such as those that block CTLA-4, and PD-1 or its ligand PD-L1 (Refs ${ }^{73,74}$ ), have been linked to new-onset autoimmune $\mathrm{T}_{1 \mathrm{DM}}{ }^{54,75,76}$. The $\mathrm{WHO}$ database of individual case safety reports described a total of 283 cases of new-onset diabetes with $>50 \%$ of patients with IClinduced diabetes mellitus presenting with $\mathrm{DKA}^{75,76}$. Additionally, a case series involving large academic medical centres estimated an incidence of $1 \%$ of new-onset T1DM with a median time of 49 days to onset and $76 \%$ of the cases presented with $\mathrm{DKA}^{74,76,77}$. 


\section{[H1] Mechanisms/pathophysiology}

In T1DM or T2DM, when there is absolute or relative insulin deficiency or in times of acute illness, which is associated with an increase in the counter-regulatory hormones, cortisol, growth hormone, glucagon and catecholamines, DKA may occur. These alterations in hormone levels and the subsequent inflammatory response form the basis of the pathophysiological mechanisms involved in DKA. The changes in hormone concentrations lead to alterations in glucose production and disposal, as well as increased lipolysis and ketone body production (Figure 2). Intercurrent illness can lead to the production of counter regulatory hormones leading to hyperglycaemia and the pro-inflammatory state resulting from an infection precipitate DKA.

\section{[H2] Gluconeogenesis and hyperglycaemia}

In diabetes mellitus, insulin deficiency leads to increased gluconeogenesis (hepatic glucose production), which is simultaneously accompanied by impaired glucose uptake and use in peripheral tissues ${ }^{78,79}$, resulting in hyperglycaemia. In healthy individuals, $\sim 20 \%$ of total endogenous glucose production also comes from the kidneys as a result of a combination of gluconeogenesis and glycogenolysis ${ }^{80}$. Endogenous renal glucose production has been speculated to be increased in DKA because data from the 1970's suggest that the presence of an acidosis increase renal glucose output, whilst impairing hepatic gluconeogenesis ${ }^{81}$. In T1DM and T2DM, increased hepatic gluconeogenesis results from the increased availability of gluconeogenic precursors such as lactate, glycerol and several gluconeogenic amino acids including alanine, glycine and serine. Furthermore, low insulin concentrations lead to catabolism of protein from muscles, liberating amino acids that are gluconeogenic and ketogenic such as tyrosine, isoleucine and phenylalanine, or purely ketogenic such as lysine and leucine. Catabolism of isoleucine, lysine and tryptophan lead to the formation of acetyl coenzyme A (acetyl CoA); catabolism of phenylalanine and tyrosine lead to the formation of acetoacetate; and leucine leads to the production of $\beta$-Hydroxy- $\beta$-methylglutaryl-CoA (HMG-CoA) - all of which feed into the production of ketone bodies. High glucagon, catecholamine and cortisol concentrations relative to insulin levels stimulate gluconeogenic enzyme activity, in particular phosphoenol pyruvate carboxykinase, fructose-1,6-bisphosphatase and pyruvate carboxylase, all of which augment hyperglycaemia ${ }^{79,82,83}$.

[H3] Ketogenesis. The increase in counter-regulatory hormone concentrations associated with 
severe insulin deficiency activates hormone-sensitive lipase in adipose tissue. Lipolysis of endogenous triglycerides by this enzyme releases large quantities of free fatty acids (FFAs) and glycerol into the circulation ${ }^{84}$. These FFAs are oxidized to ketone bodies in the hepatic mitochondria, a process mediated by high glucagon concentrations. Glucagon reduces the hepatic concentrations of malonyl CoA, which is the first committed intermediate in the lipogenic pathway ${ }^{85}$. Malonyl CoA is also a potent inhibitor of fatty acid oxidation and inhibits the enzyme, carnitine palmitoyltransferase 1 (CPT1). CPT1 regulates the uptake of FFAs into the mitochondria for $\beta$-oxidation ${ }^{86}$, causing an accumulation of acetyl CoA. Under normal circumstances, acetyl CoA enters the tricarboxylic acid (TCA) cycle (also known as Krebs cycle) and, subsequently, the mitochondrial electron transport chain to synthesize ATP. However, when acetyl CoA production exceeds the levels that can be metabolized by the TCA cycle, two molecules of acetyl CoA condense to form acetoacetyl-CoA, which can condense with another acetyl CoA molecule to form $\beta$-hydroxy- $\beta$-methylglutaryl-CoA (HMG-CoA). The enzyme HMGCoA synthase is stimulated by glucagon and inhibited by insulin, therefore, in times of fasting or insulin deprivation, the enzyme actively produces HMG-CoA. HMG-CoA within the mitochondria is lysed to form acetoacetate (as opposed to in the cytosol, where it is involved in cholesterol synthesis), which can further spontaneously degrade to form acetone or be metabolized to $\beta$ hydroxybutyrate ${ }^{87}$. The acetone, acetoacetate and $\beta$-hydroxybutyrate constitute the three ketone bodies produced by the liver. The exhaled acetone is what gives the classic 'fruity' breath in people presenting with DKA. Of these, acetoacetate and $\beta$-hydroxybutyrate are acidic, that is, they are ketoacids having pKa [G] values of 3.6 and 4.7 respectively. Concurrent with increased ketone body production, the clearance of $\beta$-hydroxybutyrate and acetoacetate is reduced. Acidosis occurs due to the buffering of the protons produced by the dissociation of ketoacids that occurs at physiological $\mathrm{pH}$. The reduced clearance of ketones contributes to the high concentration of anions in the circulation, which also contributes to the development of DKA ${ }^{88}$. However, the reason for this decreased clearance remains uncertain ${ }^{79,89}$.

Accumulation of ketoacids leads to a decrease in serum bicarbonate concentration and retention of these 'fixed acids' leads to the development of high anion gap metabolic acidosis. The anion gap is a calculation of the difference between the cations and anions in the serum and the difference can be used as a guide to the cause of the excess acidity. If there is a large difference that is not accounted for by the anions and cations in the equation, then alternative causes for the difference must be found. The most frequently used equation to calculate anion gap is $\left(\left[\mathrm{Na}^{+}\right]+\left[\mathrm{K}^{+}\right]\right)-\left(\left[\mathrm{Cl}^{-}\right]+\left[\mathrm{HCO}_{3}{ }^{-}\right]\right)$, although some investigators do not include potassium 
ion concentration owing to its negligible effect on the overall result. In healthy individuals, the reference range is most frequently $10-14 \mathrm{mmol} / /^{90-92}$. The relationship between the change in the anion gap and the change in serum bicarbonate concentration is not always $1: 1$, as was previously postulated, which might be owing to the contribution of unmeasured cations (UC) (for example, $\mathrm{Ca}^{2+}$ and $\mathrm{Mg}^{2+}$ ) and unmeasured anions (UA) (for example, $\mathrm{HPO}_{4}^{-}, \mathrm{SO}_{4}{ }^{2-}$ ). Thus, the true equation for anion gap can be expressed as $\left[\mathrm{Na}^{+}\right]+\left[\mathrm{K}^{+}\right]+\mathrm{UC}=\left[\mathrm{Cl}^{-}\right]+\left[\mathrm{HCO}_{3}^{-}\right]+\mathrm{UA}$, which can be arranged as $\left[\mathrm{Na}^{+}\right]+\left[\mathrm{K}^{+}\right]-\left[\mathrm{Cl}^{-}\right]+\left[\mathrm{HCO}_{3}{ }^{-}\right]=\mathrm{UA}-\mathrm{UC}=$ anion gap. Thus, the difference between the UAs and UCs also constitutes the anion gap ${ }^{90}$. Other components of the plasma, in particular albumin, can affect the relationship between the severity of the acidosis, the bicarbonate and anion gap and this relationship is discussed in more detail elsewhere ${ }^{90,93}$. The measure of acidity is important because as $\mathrm{pH}$ falls $<7.35$, intracellular biological systems begin to fail, leading to irreversible damage at $\sim \mathrm{pH}<6.8$. This low $\mathrm{pH}$ can lead to neurological dysfunction, leading the coma, and if severe or prolonged enough, death.

\section{[H2] Osmotic diuresis}

The severity of hyperglycaemia and the high concentrations of acetoacetate and $\beta$ hydroxybutyrate cause osmotic diuresis leading to hypovolaemia (state of extracellular volume depletion) with contraction of arterial blood volume. The osmotic diuresis also leads to a decreased glomerular filtration rate [G], therefore, reducing the ability to excrete glucose. The hypovolaemia leads to further increases in the levels of counter-regulatory hormones, further aggravating hyperglycaemia ${ }^{94}$. The resulting low circulating volume leads to generalised hypoperfusion and can also lead to a rise in lactic acid. Owing to lack of perfusion, peripheral tissues become deprived of oxygen and switch to anaerobic respiration, thereby generating lactate, worsening the acidaemia (the state of low blood $\mathrm{pH}$ ). The lack of renal perfusion can lead to pre-renal renal failure. This lack of renal perfusion means that there is an inability to adequately excrete acids such as sulphate, phosphate or urate, further exacerbating the high anion gap acidaemia. The osmotic diuresis, as well as the associated vomiting and inability to take fluid orally or a lower conscious level lead to worsening of the dehydration. The hyperglycaemia might be worsened by the ingestion of sugar sweetened beverages to quench the thirst experienced by these individuals.

\section{[H2] Electrolyte disturbance}

Insulin maintains the potassium (a predominantly intracellular cation) concentrations within the intracellular fluid. Thus, the lack of insulin causes potassium to move into the extracellular 
space. As the plasma $\mathrm{pH}$ falls due to the rise in ketone concentrations, plasma bicarbonate ions act as one of the main buffers to maintain the physiological $\mathrm{pH}$ (that is, $\mathrm{pH}$ 7.4). As acidaemia progresses and the $\mathrm{pH}$ falls further, the bicarbonate concentration drops because it buffers [G] the increase in hydrogen ion concentration, and further tissue buffering becomes crucial. To achieve this, extracellular hydrogen ions from the ketoacids are exchanged for intracellular potassium ions. In addition, the extracellular hypertonicity [G] causes movement of water from the intracellular space to the extracellular space leading to further loss of intracellular potassium. Furthermore, owing to the osmotic diuresis, the circulating volume decreases and aldosterone concentration increases. Aldosterone works by conserving sodium reabsorption in the kidney by excreting potassium in the urine, leading to further potassium loss. The end effect of these physiological attempts at maintaining buffering capacity and electrical neutrality is hyperkalaemia. A study from 1956 showed that for each 0.1 unit fall in $\mathrm{pH}$, serum potassium concentration increased by $0.6 \mathrm{mmol} / \mathrm{I}^{95}$. Thus in the acute stage before fluid and insulin treatment is started, serum potassium can be as high as $\geq 7.0 \mathrm{mmol} / \mathrm{l}$, yet because of the renal loss, total body potassium stores are usually substantially depleted, which is estimated to be 3$5 \mathrm{mmol} / \mathrm{Kg}^{9}$.

\section{[H2] Inflammation}

Severe hyperglycaemia and the occurrence of ketoacidosis result in a pro-inflammatory state, evidenced by an elevation of oxidative stress markers and increased concentrations of proinflammatory cytokines ${ }^{96-99}$. This increase in inflammatory cytokines leads to white adipose tissue dysfunction by inhibiting insulin signalling or increasing lipolysis, thereby leading to greater transport of FFAs to the liver, which act as ketogenic substrates ${ }^{100-102}$. In diabetic conditions, impaired insulin signalling that results in severe hyperglycaemia can induce the liver to produce CRP (a pro-inflammatory marker) under the influence of activated macrophages that secrete pro-inflammatory cytokines such as, IL-6, IL-1 $\beta$, and TNF. These cytokines, in turn, can impair insulin secretion and reduce insulin action further exacerbating $D K A^{97,98,103,104}$. The elevated FFAs also induce insulin resistance and at the same time cause endothelial dysfunction by impairing nitric oxide production in endothelial cells ${ }^{105,106}$. Together, the inflammatory response induces oxidative stress and the subsequent generation of reactive oxygen species lead to capillary endothelial disruption and damage of cellular lipids, proteins, membranes, and DNA ${ }^{97,99}$. The inflammatory state caused by has also been hypothesized to be involved in causing complications of DKA in children, particularly cerebral oedema and cerebral injury ${ }^{107-109}$. The cerebral oedema in DKA is vasogenic (that is, resulting from the disruption of 
the blood-brain barrier) but the mechanism remains undetermined.

The reasons for coma or reduction in cognitive ability in DKA are yet to be elucidated. Given that some people are fully alert and orientated with a $\mathrm{pH}$ of 6.9 , whereas others are obtunded at a pH of 7.2 suggests that an element of 'physiological reserve' might be involved. However, the degree of circulatory volume depletion, high glucose concentrations and rapid shift of electrolytes between the intracellular and extracellular spaces might also play a part.

\section{[H2] SGLT2 inhibitor-induced ketoacidosis}

By promoting a glycosuria, the SGLT2 inhibitors lower circulating glucose concentrations ${ }^{110}$. As glucose concentrations drop, insulin concentrations also drop and glucagon rises. Together these changes promote lipid $\beta$-oxidation, and ketoacid production occurs ${ }^{111-113}$. In patients already using insulin, as glucose concentrations drop, insulin doses may be reduced, but ketogenesis is not prevented. As ketone concentrations continue to rise, DKA may occur - but crucially, as the circulating glucose concentrations are low, euglycaemic DKA occurs more frequently in these individuals ${ }^{114,115}$. The mechanism for the development of DKA with SGLT2 inhibitors has been discussed in detail elsewhere ${ }^{114,115}$.

\section{[H2] Alcoholic ketoacidosis}

Alcoholic ketoacidosis has a different pathogenesis from DKA and develops in people with chronic alcohol abuse who have binged, resulting in nausea, vomiting and acute starvation ${ }^{116,117}$. Blood glucose concentration is the key diagnostic feature that differentiates DKA and alcohol-induced ketoacidosis. Acute alcohol withdrawal can cause counter-regulatory hormone release and any accompanying starvation will be associated with low insulin secretion, which, in turn, causes lipolysis and ketogenesis. Furthermore, the enzyme, alcohol dehydrogenase, metabolizes ethanol to acetaldehyde, which is metabolized to acetic acid and transported into the mitochondria, where it is converted into acetyl CoA that subsequently condenses to acetoacetate ${ }^{118}$. In contrast to DKA that usually presents with severe hyperglycaemia, the presence of ketoacidosis without hyperglycaemia in an alcoholic patient is virtually diagnostic of alcoholic ketoacidosis ${ }^{117,119}$.

\section{[H2] Starvation ketosis}

Starvation ketosis occurs when a person has a prolonged reduced calorie intake of 
$<500 \mathrm{Kcal} / \mathrm{day}^{120}$. With little or no carbohydrate intake, insulin secretion is decreased, leading to lipolysis and ketogenesis. However, starvation ketosis differs from DKA; in healthy individuals or in individuals with obesity without diabetes who starve, $\beta$-hydroxybutyrate concentrations can reach $5-6 \mathrm{mmol} / \mathrm{l}$, but this takes several days of absolute starvation with almost very little or no caloric intake ${ }^{121,122}$, or $4-5 \mathrm{mmol} / \mathrm{l}$ after 10 days of starvation ${ }^{123}$. For comparison, in a healthy, non-starving individual, $\beta$-hydroxybutyrate concentrations should be $<0.3 \mathrm{mmol} / 1$. An individual is able to adapt to prolonged fasting by increasing brain and muscle ketone clearance as well as renal compensation by increasing acid excretion, in particular ammonia ${ }^{121,124}$. As this condition develops over many days, electrolyte imbalance (for example, low bicarbonate concentrations) is less likely to occur due to the ability of the kidney to compensate. However, if electrolyte intake is also limited, then eventually electrolyte disturbances will occur ${ }^{124}$.. Thus, as a result of renal compensation, starvation-induced ketosis is unlikely to present with a serum bicarbonate concentration $<18.0 \mathrm{mmol} / \mathrm{L}^{120}$. This serum bicarbonate corresponds to a mean $B-$ hydroxybutyrate concentration of $5.68( \pm 1.5) \mathrm{mmol} / \mathrm{l}$ in the UK national survey of DKA; it is likely that it took only a few hours of insulin deprivation to achieve that ketone concentration in patients with $D K A^{35}$.

\section{[H1] Diagnosis, screening and prevention}

\section{[H2] Presentation}

DKA frequently presents with a short history, with symptoms developing usually over a few hours. These include the classic symptoms of hyperglycaemia - polyuria (excessive urine production), polydipsia (excessive thirst) and, in those for whom DKA is the first presentation of diabetes, weight loss (Figure 3). Polyphagia (excessive hunger) has been reported in children, but remains rare in adults $^{125}$ Gastrointestinal symptoms such as nausea, vomiting and generalized abdominal pain are reported in $>60 \%$ of patients ${ }^{1,126}$. Abdominal pain, sometimes mimicking an acute abdomen, is especially common in children and in patients with severe metabolic acidosis. Abdominal pain typically resolves during the first 24 hours of treatment and lack of resolution of abdominal pain within this time frame should prompt a search for other causes ${ }^{126}$. Although the cause of the gastrointestinal complaints has not been fully elucidated, delayed gastric emptying, ileus (that is, lack of movement in the intestines that leads to a delay in transit), electrolyte disturbances and metabolic acidosis have been implicated ${ }^{1,126}$. 
Physical examination of adults and children usually reveals signs of circulatory volume depletion, including dry mucous membranes and tachycardia. Mental status on admission varies from full alertness to lethargy and stupor, with $<20 \%$ of adults hospitalized showing loss of consciousness $^{127}$. As $\mathrm{pH}$ drops, respiratory compensation for the metabolic acidosis, that is, excreting acidic carbon dioxide in an attempt to maintain plasma $\mathrm{pH}$, leads to Kussmaul respirations (a deep and laboured breathing pattern) in individuals with DKA and the breath might have a classic fruity odour owing to acetone exhalation. Most adults and children are normothermic or even hypothermic at presentation even in the presence of infection. Hypotension might be observed in adults but is rarely present in children. In fact, for reasons unknown, studies have documented a high frequency of hypertension in children with DKA, in spite of substantial volume depletion ${ }^{128}$. Therefore, it is important not to rely on blood pressure as a marker of DKA severity in children.

\section{[H2] Diagnosis}

The diagnosis of DKA is based on the triad of hyperglycaemia, ketosis and metabolic acidosis $^{129}$. Although the ADA, Joint British Diabetes Societies and the International Society of Pediatric and Adolescent Diabetes agree that the main diagnostic feature of DKA is the elevation in circulating total blood ketone concentration, the other diagnostic criteria such as serum glucose and bicarbonate concentrations differ(Table 1 $)^{8,9,52,130}$. Studies have shown that between $3-8.7 \%$ of adults who present with DKA have normal or only mildly elevated glucose concentrations $(<13.9 \mathrm{mmol} / \mathrm{l}[250 \mathrm{mg} / \mathrm{d}]])-$ a condition known as euglycaemic DKA ${ }^{131-133}$. Euglycaemic DKA has been reported during prolonged starvation, with excessive alcohol intake, in partially treated individuals (i.e. those receiving inadequate doses of insulin), during pregnancy and in those who use an SGLT-2 inhibitor ${ }^{65,133,134}$. In those taking SGLT-2 inhibitors who may present with DKA but without severe hyperglycaemia, a thorough medication history is key to confirming the diagnosis.

When individuals present with euglycaemic DKA, the admission biochemistry is relatively nonspecific and might be affected by the degree of respiratory compensation, the coexistence of a mixed acid-base disturbance or other comorbidities ${ }^{116}$. Studies from the 1980 s documented high anion gap acidosis in $46 \%$ of people (14-55 years of age) admitted for DKA, whilst $43 \%$ had mixed anion gap acidosis and hyperchloraemic metabolic acidosis, and $11 \%$ develop hyperchloraemic metabolic acidosis ${ }^{135}$, however, current data do not describe patterns of 
acidosis on admission and these differing categories have no impact on the diagnosis or immediate treatment of DKA. The fact that not all people fall into a single category indicated the heterogeneity of the biochemical abnormalities observed in DKA. The hyperchloraemic metabolic acidosis is most frequently observed in those given large volumes of $0.9 \%$ sodium chloride solution, during the recovery phase of the admission ${ }^{136}$.

Assessment of ketonaemia (that is, blood ketone concentration) can be performed by the nitroprusside reaction in urine or serum or by direct measurement of $\beta$-hydroxybutyrate in capillary blood using point-of-care testing or by the hospital laboratory ${ }^{8,88}$. Although easy to perform, the nitroprusside test measures acetoacetate and does not detect $\beta$-hydroxybutyrate, the main ketone in $\mathrm{DKA}^{79,137}$. As plasma or urine acetoacetate concentration only accounts for $15-40 \%$ of the total ketone concentration, relying on acetoacetate using urine ketone testing alone is likely to underestimate the severity of ketonaemia ${ }^{52,138}$. In addition, several sulfhydryl drugs (for example, captopril) or medications such as valproate that are taken for comorbidities including hypertension or epilepsy, give false-positive nitroprusside urine tests ${ }^{52,87}$. Using expired or improperly stored test strips can give false-negative results, which can also occur when urine specimens are highly acidic, for example, after the consumption of large amounts of vitamin $C^{87}$. In addition, unlike the ADA guidelines, the Joint British Diabetes Societies strongly discourages the use of urinary ketone tests ${ }^{8,88}$ and recommends direct measurement of $\beta$ hydroxybutyrate from a blood sample to assess ketonaemia in ambulatory and hospital care. A more detailed explanation of the differences of urinary and plasma ketone tests can be found elsewhere ${ }^{88}$.

Studies in adults and children with DKA have reported a good correlation between $\beta$ hydroxybutyrate and the severity of acidaemia measured from serum bicarbonate concentration $^{139,140}$. A bicarbonate concentration of 18.0 and $15.0 \mathrm{mmol} / \mathrm{L}$ corresponds to 3.0 and $4.4 \mathrm{mmol} / \mathrm{L}$ of $\beta$-hydroxybutyrate, respectively, suggesting that when plasma ketone tests are unavailable, a 'best guess' can be made according to the bicarbonate concentration. Measurement of $\beta$-hydroxybutyrate can also guide response to treatment. The UK guidelines recommends to intensify the treatment if the plasma concentration of $\beta$-hydroxybutyrate does not decrease by $0.5 \mathrm{mmol} / /$ per hour following fluid and intravenous insulin administration ${ }^{130}$.

Many individuals with hyperglycaemic crises present with combined features of DKA and HHS (Box 1). Previous work has reported that among 1,211 patients who had a first admission with 
hyperglycaemic crises criteria based on the ADA guidelines ${ }^{8}, 465$ (38\%) had isolated DKA, 421 (35\%) had isolated HHS, and $325(27 \%)$ had combined features of DKA and HHS. After adjustment for age, sex, BMI, ethnicity and Charlson Comorbidity Index score (which predicts the 1-year mortality of a patient with a range of comorbidities) with combined DKA-HHS had higher in-hospital mortality compared with patients with isolated DKA (adjusted OR 2.7; 95\% CI $1.4-4.9)^{141}$.

\section{[H2] Systemic assessment}

Upon hospital admission, immediate assessment of the haemodynamic state and level of consciousness, together with measurement of blood glucose, blood or urine ketones, serum electrolytes, venous blood gases and complete blood count should be performed. As part of the rapid assessment of the individual, precipitants for DKA should be sought, including an ECG to exclude acute coronary syndrome and repolarization abnormalities (that is, peaked $\mathrm{T}$ waves) due to hyperkalaemia.

The systemic effect of DKA in adults depends on the severity of the acidaemia and circulatory volume depletion (Table 1). However, one of the drawbacks of the ADA classification is that the degree of acidaemia is imperfectly correlated with the patient's level of consciousness ${ }^{8}$. Thus, it is unclear whether a patient who presents with a $\mathrm{pH}$ of $<7.0$, yet is fully conscious, or another who presents comatose with a $\mathrm{pH}$ of 7.26 are mild or severe. Other markers of severity including ketone concentrations $(>6.0 \mathrm{mmol} / \mathrm{l})$, venous $\mathrm{pH}<7.0$, hypokalaemia on admission $(<3.5 \mathrm{mmol} / \mathrm{l})$, systolic blood pressure $(<90 \mathrm{mmHg})$, pulse rate (either $>100 \mathrm{bpm}$ or $<60 \mathrm{bpm})$, oxygen saturations ( $<92 \%$, assuming it is normal at baseline), and Glasgow Coma Scale Score $(<12)$ have been suggested by the UK guideline ${ }^{130}$. The Glasgow Coma Scale comprises subscale scores for behaviours (such as eye opening and verbal and motor responses to stimuli), with a higher total score indicating a higher level of consciousness of the patient $)^{142}$. If breathing is compromised due to lethargy or coma, then urgent airway management needs to be initiated with support of the intensive care team.

In adults, mortality is often due to the underlying precipitant such as infection or intercurrent illness. However, lack of access to treatment might be the cause of excess mortality in lowresource environments. In children, mortality resulting from DKA is mainly the result of cerebral oedema or cerebral injury. Thus, assessment of consciousness level is of particular importance. 


\section{[H2] Prevention}

In individuals with known diabetes, prevention of DKA and hospital admission is feasible. 'Sick day rules' are a simple set of instructions that patients can follow when they are unwell for any reason. These rules state that - particularly in those with T1DM, insulin must never be stopped, even if the individuals do not consume solids or fluids ${ }^{143}$. Also, when unwell, blood glucose concentrations should be measured every few hours and blood or urine ketone concentrations should be measured at least twice a day. If ketones are detected, increased insulin doses should be administered. Maintaining good hydration is also important. If vomiting due to illness is persistent, then hospital admission is often necessary. One study reported that telephone consultations with nurses or diabetes educators can help prevent DKA admissions ${ }^{144}$.

\section{[H1] Management}

Most of the data regarding management of DKA come from North America, Europe and Australia. Data from other parts of the world show a lack of accessibility of treatments. Individuals living in areas of low socio-economic status have no or limited access to insulin owing to an inability to main 'security of supply'145. Many studies have shown that in parts of Africa, DKA was the main cause of death in people who require insulin who were admitted to hospital ${ }^{41,146}$.

Insulin therapy and fluid and electrolyte replacement are the cornerstones of DKA treatment. The aim is to correct acidaemia, restore normal circulatory volume and normalize blood glucose concentrations and acid-base disturbances to restore normal levels of inflammatory and oxidative stress markers ${ }^{106,147}$.

Careful monitoring of the patient's response to DKA treatment and appropriate adjustments in treatment based on this response are essential. Monitoring should include tracking of blood pressure, pulse and respiratory rate as well as accurate documentation of fluid intake and output. For most patients, glucose levels should be monitored hourly and electrolytes (sodium, potassium, chloride and bicarbonate), urea nitrogen, creatinine and venous $\mathrm{pH}$ should be measured every 2-4 hours. Levels of phosphate, calcium and magnesium are measured less frequently (generally every 4-6 hours). Neurological status should be monitored hourly using the Glasgow Coma Scale ${ }^{142}$ or similar assessments, for example, AVPU (Alert, Voice, Pain, Unresponsive) scale $^{148}$. More frequent monitoring (that is, every 30 minutes) might be 
necessary for children with DKA and impaired cognitive status. There should be a low threshold for moving individuals presenting with altered cognitive status or severe metabolic derangement and those who fail to improve after initial treatment to an intermediate care unit (high dependency) or critical care unit in the hospital ${ }^{1,149}$. Alternatively, people with the ADA-classified mild DKA (Table 1) who have normal cognition and are able to eat and drink can be treated with oral fluids and subcutaneous insulin in an acute care setting, potentially avoiding hospitalization $^{1,149}$.

The criteria for the resolution of a DKA episode should be a combination of a blood glucose of $<200 \mathrm{mg} / \mathrm{dL}(11.1 \mathrm{mmol} / \mathrm{l})$, a serum bicarbonate level of $\geq 18.0 \mathrm{mmol} / \mathrm{l}$, a venous $\mathrm{pH}>7.30$ and a calculated anion gap of $\leq 14.0 \mathrm{mmol} / \mathrm{I}^{8}$. A serum $\beta$-hydroxybutyrate $<1.0 \mathrm{mmol} / \mathrm{l}$ can also be used to determine resolution of DKA. In settings where $\beta$-hydroxybutyrate measurements are unavailable, normalization of the anion gap is the best indicator of DKA resolution ${ }^{8}$.

\section{[H2] Volume correction}

Administration of intravenous fluid is the key to intravascular volume correction, thereby improving renal perfusion. The concomitant decrease in circulating counter-regulatory hormone concentrations also reduces insulin resistance ${ }^{150}$. In adults with DKA, the ADA and UK guidelines recommend normal saline ( $0.9 \%$ sodium chloride solution) for the initial fluid replacement ${ }^{8,130}$, administered at an initial rate of 500-1000 ml/hour during the first 2-4 hours. In an attempt to understand the best resuscitation fluid to use in DKA, a study comparing intravenous infusion of normal saline with Ringer's lactate (a mixture of sodium chloride, sodium lactate, potassium chloride and calcium chloride) found no difference in the time to resolution of DKA, although hyperglycaemia resolved later in the Ringer's lactate group ${ }^{151,152}$. A potential 'trap' for the unwary is the development of hyperchloraemic metabolic acidosis owing to excessive chloride resulting from the administration of high volumes of saline. This is because $0.9 \%$ saline contains a higher concentration of chloride ions than serum $(154 \mathrm{mmol} / \mathrm{l}$ compared with $100 \mathrm{mmol} / /)^{9}$. Although there are generally no acute adverse effects of hyperchloraemic metabolic acidosis, the development of hyperchloraemic metabolic acidosis can delay transition to subcutaneous insulin treatment if the serum bicarbonate concentration is used as an indicator of DKA resolution. After restoration of intravascular volume, the serum sodium concentration and state of hydration assessed by blood pressure, heart rate and fluid balance should determine whether the rate of normal saline infusion can be reduced to $250 \mathrm{ml} /$ hour or changed to $0.45 \%$ sodium chloride $(250-500 \mathrm{ml} / \mathrm{h})^{8}$. A study has proposed different approaches for 
individualizing fluid treatment based on calculations of sodium and fluid deficits ${ }^{153}$. Plasma glucose concentrations typically decrease to $<200 \mathrm{mg} / \mathrm{dl}(11.1 \mathrm{mmol} / \mathrm{l})$ before ketoacidosis resolves. Thus, once the plasma glucose concentration is $\sim 200 \mathrm{mg} / \mathrm{dL}(11.1 \mathrm{mmol} / \mathrm{L})$, the replacement fluids should contain $5-10 \%$ dextrose (to prevent hypoglycaemia) to allow continued insulin administration until ketonaemia is corrected ${ }^{1}$..

In children ( $<18$ years of age) with DKA, fluid deficits can vary between 30 and $100 \mathrm{ml} / \mathrm{Kg}$, depending on the duration of symptoms and ability to maintain hydration. Clinical assessments (using capillary refill time, skin turgor and other aspects of the physical exam) to estimate the degree of fluid deficit are frequently inaccurate in children with DKA ${ }^{154-156}$, therefore, average fluid deficits of $\sim 70 \mathrm{ml} / \mathrm{Kg}$ should be assumed for most children. An initial bolus of $10-20 \mathrm{ml} / \mathrm{Kg}$ of $0.9 \%$ normal saline or other isotonic fluid should be administered promptly over 30-60 minutes to help restore organ perfusion. In children with hypovolaemic shock, the initial fluid administration should be $20 \mathrm{ml} / \mathrm{kg}$ over 15-30 minutes. Fluid boluses can be repeated if necessary based on the haemodynamic state. Such bolus fluid administration is preferred in children to ensure more rapid tissue perfusion than can be achieved than by slower continuous fluid infusion. Following the initial fluid bolus, the remaining fluid deficit should be replaced over 24-48 hours, using $0.45-0.9 \%$ sodium chloride. In the 1980 s and early 1990s, slower administration of intravenous fluids was recommended in paediatric patients with DKA to prevent cerebral oedema ${ }^{157,158}$. A large RCT (the Pediatric Emergency Care Applied Research Network FLUID Study), however, found no differences in acute or post-recovery neurological outcomes in children with DKA treated with rapid versus slower volume correction ${ }^{159}$ or between the use of $0.9 \%$ versus $0.45 \%$ sodium chloride. In a sub-analysis involving children with severe acidosis and cognitive impairment resulted in improved mental status during DKA treatment ${ }^{159}$. These findings are reassuring as they assure that variations in fluid treatment protocols are not the cause of cerebral oedema or cerebral injury during DKA.

In both adult and paediatric DKA, the 'two bag' method of fluid replacement is often used, whereby two concurrent bags of fluid are used. Although both bags have identical electrolyte content $(0.45 \%$ or $0.9 \%$ saline with potassium), only one bag contains $10 \%$ dextrose. The bag without dextrose is used initially as the resuscitation fluid and the dextrose infusion is added when the glucose drops to $200-250 \mathrm{mg} / \mathrm{dl}(11.0-13.9 \mathrm{mmol} / \mathrm{l})$. The two bag method prevents the need to continually change infusion fluids according to glucose concentrations ${ }^{160-162}$. 
The measured serum sodium concentration at presentation reflects relative losses of sodium and extracellular free water as well as the osmotic effect of hyperglycaemia. Most adults and children with DKA have mild hyponatraemia at presentation, which gradually returns to the normal range of $135-145 \mathrm{mmol} / \mathrm{l}$ as blood glucose levels decline and water moves back into intracellular space. The measured sodium concentration has been proposed to decline by $1.6 \mathrm{mmol} / \mathrm{l}$ for every $100 \mathrm{mg} / \mathrm{dl}(5.5 \mathrm{mmol} / \mathrm{L})$ rise in the serum glucose concentration above the normal range such that a 'corrected' sodium concentration can be calculated as the measured serum sodium concentration $+1.6 \times[$ (glucose concentration in $\mathrm{mg} / \mathrm{dL}-100) / 100]$. This theoretically determined correction factor was found to correlate well with empirical data from a study of children with DKA ${ }^{163}$ that enables a better assessment of sodium deficit (and therefore, requirements for replacement) can be made. Alternative correction factors have also been proposed and tracking the corrected sodium concentration during treatment can be useful for monitoring the adequacy of relative rates of fluid and sodium administration ${ }^{164,165}$.

\section{[H2] Insulin administration}

Most people with DKA will be treated initially with an intravenous insulin infusion until the DKA has resolved and the patients are eating and drinking normally, at which time they will be transferred to subcutaneous insulin.

[H3] Intravenous infusion. In most adults with DKA, a continuous intravenous infusion of regular (soluble) insulin is the treatment of choice. In many hospitals, the intravenous fluids are administered whilst the intravenous insulin infusion is being prepared ${ }^{35}$. In adults, many treatment protocols recommend the administration of insulin ( 0.1 unit per kg body weight) bolus intravenously or intramuscularly if a delay in getting venous access is anticipated, which is immediately followed by fixed rate intravenous insulin infusion at 0.1 unit/kg/hour. Once the blood glucose concentration is $\sim 200 \mathrm{mg} / \mathrm{dl}(11.0 \mathrm{mmol} / \mathrm{l})$ the insulin infusion rate is adjusted to between $0.02-0.05$ units $/ \mathrm{kg} / \mathrm{hour}$ and an of $5 \%$ dextrose is added to the infusion, to maintain glucose concentrations at $140-200 \mathrm{mg} / \mathrm{dL}(7.8-11.0 \mathrm{mmol} / \mathrm{l})$ until resolution of ketoacidosis ${ }^{8}$.

For treatment of DKA in children, the International Society for Pediatric and Adolescent Diabetes (ISPAD) guidelines recommend intravenous administration of regular insulin as a continuous infusion at $0.1 \mathrm{units} / \mathrm{kg} / \mathrm{hour}^{22}$, which should be started immediately after the initial intravenous fluid bolus(es). Intravascular volume expansion before insulin administration is particularly important in children who present with very high glucose levels and hyperosmolality 
because intravascular volume will decline substantially as the hyperosmolar state resolves. An initial bolus of insulin is not necessary as continuous intravenous insulin infusion rapidly achieves steady state serum insulin levels ${ }^{166,167}$. A few small studies reported that insulin infused at $0.05 \mathrm{unit} / \mathrm{kg} /$ hour can resolve hyperglycaemia over a similar time frame compared with the standard dosage of 0.1 units $/ \mathrm{kg} / \mathrm{hour}^{168-170}$. This lower dosage might be considered for very young children ( $<6$ years old) or others with greater insulin sensitivity for whom the standard dosage might not be necessary ${ }^{168}$. In general, intravenous insulin is recommended for treating children with DKA due to unreliable subcutaneous insulin absorption in the volume-depleted state. However, subcutaneous administration can be used in children with mild DKA (Table 1)or in situations when intravenous administration is not possible. When the serum glucose concentration decreases to $\sim 250 \mathrm{mg} / \mathrm{dL}(13.9 \mathrm{mmol} / \mathrm{L})$, intravenous fluids containing dextrose should be used to maintain the serum glucose concentration at $\sim 100-150 \mathrm{mg} / \mathrm{dl}$ (5.5 to $8.3 \mathrm{mmol} / \mathrm{l})$ while maintaining the total fluid infusion rate ${ }^{22}$.

\section{[H3] Maintenance insulin therapy.}

Once biochemical resolution of DKA is achieved and the patient is eating and drinking normally, subcutaneous insulin therapy can be started in adults as well as children. Adults with newly diagnosed diabetes mellitus or those who have not previously received insulin should be started on total insulin dosage of $0.5-0.6$ units $/ \mathrm{kg} /$ day. Patients who were already on subcutaneous insulin prior to DKA admission should resume their previous insulin regimens.

For most adults, a basal bolus regimen (that is, rapid-acting insulin given with each meal as well as a once or twice daily administered long-acting basal insulin) is preferred over the use of regular insulin because of the lower rate of in-hospital hypoglycaemia despite similar glucose control ${ }^{171}$. In children, insulin regimens differ depending on the centre; however, basal-bolus regimens are generally preferred. Previous work has shown that the administration of frequent doses of subcutaneous rapid-acting insulin analogues (given every 1-2 hours), can be an acceptable alternative to an intravenous insulin infusion as both treatments resolve DKA in similar time ${ }^{172-174}$. In adults and children, subcutaneous rapid-acting insulin is given as a bolus of $0.2 \mathrm{unit} / \mathrm{kg}$ at the start of treatment, followed by $0.1-0.2 \mathrm{unit} / \mathrm{kg}$ every 1-3 hours until the blood glucose concentration is $<250 \mathrm{mg} / \mathrm{dl}(13.9 \mathrm{mmol} / \mathrm{l})$, then the dose is reduced by half and continued every 1-2 hours until resolution of $D K A^{172,175}$. The total insulin daily dose is generally $0.7-0.8 \mathrm{unit} / \mathrm{Kg} /$ day in the prepubertal child and 1.0-1.2unit/Kg/day in the pubertal adolescent ${ }^{176}$. 
Clinical trials and meta-analyses that compared continuous subcutaneous insulin infusion (CSII) with discrete subcutaneous insulin doses (for example, basal bolus regimens) have shown small but significant reductions in $\mathrm{HbA1c}$ and risk of severe hypoglycaemia in those receiving CSII. In addition, these studies have found an increased risk of developing ketoacidosis with CSII primarily due to device malfunction and/or catheter occlusion ${ }^{177-179}$, a finding confirmed by the UK National Diabetes Pump Audit ${ }^{60}$. However, the use of frequent home glucose monitoring has reduced this complication considerably ${ }^{178}$. In adults and children, intramuscular administration of rapid-acting insulin is also effective. However, this route is more painful than subcutaneous injections and potentially would be contraindicated in those taking anticoagulants ${ }^{1,180,181}$.

\section{[H2] Potassium replacement}

Nearly all patients with DKA have substantial potassium deficits at the time of presentation and potassium replacement is almost always required (Box 2). At presentation, serum potassium concentrations are frequently normal or slightly elevated in spite of total body deficits. As insulin treatment starts, ketone production is suppressed, and the acidosis begins to resolve. In addition, insulin drives potassium back into the cell, and the individual can become profoundly hypokalaemic. Hypokalaemia occurs frequently despite aggressive potassium replacement ${ }^{35,141}$ and frequent monitoring of potassium during the first few hours of treatment is an essential part of managing $\mathrm{DKA}^{8,130}$. Because of potentially rapid shifts in potassium and the possible risk of developing cardiac arrhythmias, continuous cardiac monitoring is recommended in all cases where potassium is being administered at $>10 \mathrm{mmol} / \mathrm{hr}$.

Two studies showed that within 24-48 hours of admission, potassium levels declined on average from $4.8 \pm 1.0$ and $4.9 \pm 1.1$ to $3.65( \pm 0.66)$ and $3.66( \pm 0.6) \mathrm{mmol} / \mathrm{l}$, respectively, among adults with $\mathrm{DKA}^{35,141}$. The development of severe hypokalaemia $(<2.5 \mathrm{mmol} / \mathrm{l})$ was associated with increased mortality (OR 3.17; 95\% Cl 1.49-6.76) ${ }^{141}$. The association between hypokalaemia within 48 hours and mortality remained significant after adjusting for demographic variables and metabolic parameters on admission suggesting that hypokalaemia is most likely the cause of increased mortality and not any other confounding factors.

In patients who develop symptomatic hypokalaemia (muscle weakness and cardiac arrhythmia), potassium replacement should be started and insulin administration should be delayed until the potassium concentration has risen to $>3.3 \mathrm{mmol} / \mathrm{l}$. A survey of the management of DKA in the 
UK showed that an intravenous insulin infusion rate of $0.1 \mathrm{unit} / \mathrm{Kg} / \mathrm{hour}$ was associated with $55 \%$ of adults developing hypokalaemia ${ }^{35}$. Although no harm was associated with this hypokalaemia, it provides support for the practice of reducing the insulin infusion rate to $0.05 \mathrm{unit} / \mathrm{Kg} / \mathrm{hr}$ after glucose levels decline.

Similar to adults, hypokalaemia is rarely present in children before DKA treatment. In these rare cases, earlier and more aggressive potassium replacement is necessary and the insulin infusion should be delayed until urine output is documented and serum potassium has been restored to a near normal concentration ${ }^{22}$. Serum potassium levels should be monitored every $2-4$ hours and the potassium concentration in intravenous fluids adjusted to maintain normal potassium levels. A cardiac monitor or frequent ECGs should be considered during intravenous potassium replacement.

The choice of potassium salts to use for replacement has been a subject of debate. Adult protocols typically recommend potassium chloride alone, but paediatric protocols often recommend using a mixture of potassium chloride and potassium phosphate or potassium acetate $^{22}$ to reduce the chloride load thereby diminishing the risk of hyperchloraemic acidosis.

\section{[H2] Bicarbonate administration}

Treatment with intravenous bicarbonate is not routinely recommended for adults or children with DKA. Time to biochemical resolution, length of hospitalisation or mortality have not been shown to improve with bicarbonate treatment ${ }^{182-185}$. Bicarbonate therapy might increase the risk of hypokalaemia, slow the resolution of ketosis, cause paradoxical increases in cerebral acidaemia due to an increase in tissue $\mathrm{pCO}_{2}$ and increase the risk of cerebral injury ${ }^{186,187}$. Some commentaries have suggested that specific subsets of adults with DKA might benefit from bicarbonate administration, however, data from randomized trials are lacking ${ }^{93}$.

\section{[H2] Phosphate replacement}

Similar to potassium, serum phosphate concentrations are typically normal at presentation but intracellular depletion is present and serum concentrations decline during DKA treatment. Phosphate replacement is necessary in those with serum phosphate concentration $<1.0$ $1.5 \mathrm{mg} / \mathrm{dl}(0.3-0.5 \mathrm{mmol} /)^{8}$. Inclusion of phosphate in the infusion has been proposed to diminish the risk of hypophosphataemia, which has been associated with severe complications in some patients including rhabdomyolysis (breakdown of skeletal muscles), renal failure, respiratory 
failure, arrhythmias and haemolytic anaemia ${ }^{98,188-191}$. Thus, for individuals with cardiac dysfunction, anaemia or respiratory depression, phosphate replacement should be strongly considered. Concern over phosphate replacement mainly centres on an increased risk of hypocalcaemia; however, studies documenting hypocalcaemia with phosphate replacement used more aggressive phosphate replacement than recommended in current protocols ${ }^{192}$. Studies in the 1980s found increases in red blood cell 2,3-disphosphoglycerate (DPG, which liberates oxygen from haemoglobin in peripheral tissues) levels with phosphate replacement but did not detect any beneficial effect of phosphate replacement on clinical outcomes ${ }^{193,194}$. The sample size for these studies, however, was very small and statistical power to detect differences in outcomes was very limited. Phosphate levels should be monitored during treatment at least every $4-6$ hours, although more frequent monitoring (every 2-3 hours) is recommended for those not receiving phosphate replacement.

\section{[H2] Cerebral injury}

Among the severe complications of DKA, cerebral injury is the most well recognized (Table 3). Although rare in adults, severe cerebral injury occurs in $0.3-0.9 \%$ of DKA episodes in children ${ }^{186,195,196}$ and is associated with high rates of mortality $(21-24 \%)$ and permanent neurological morbidity $(20-26 \%)^{186,195,196}$. Risk factors for cerebral injury include severe acidaemia and severe deficits in circulatory volume ${ }^{186,195,196}$. Younger children ( $<5$ years) are at greater risk for DKA-related cerebral injury, reflecting the greater severity of DKA at presentation in this age group in whom symptoms of diabetes can be less apparent and $\beta$-cell destruction is often aggressive. Although severe cerebral injury occurs in $<1 \%$ of children with DKA, mild cerebral injury occurs much more commonly - possibly in the majority of children ${ }^{197,198}$. Subtle deficits in memory, attention and intelligence quotient have been reported in children with T1DM with a history of DKA compared with children with T1DM without DKA history ${ }^{199-201}$. These differences persist after adjusting for HbA1cand demographic factors. Microstructural and macrostructural alterations, such as increased total white matter volume and other changes in the in the frontal, temporal, and parietal white matter in the brain have also been associated with DKA in children ${ }^{199}$.

Cerebral injury can exist at the time of presentation, before starting treatment, but is more common during the first 12 hours of treatment ${ }^{186,196,202}$. Changes in mental status, onset of headache during DKA treatment and recurrence of vomiting are indicative of cerebral injury ${ }^{203}$. Cerebral oedema may be found on imaging studies, but many individuals have no detectable 
imaging abnormalities at the time of neurological deterioration, suggesting that cerebral oedema and/or infarction can develop hours or days after treatment has started ${ }^{203}$. For this reason, treatment for DKA-related cerebral injury should not be delayed while awaiting imaging studies. Treatment involves administration of mannitol or hypertonic saline, both of which induce osmotic shifts of fluid from within the intracellular space into the vascular compartment.

\section{[H2] The precipitating illness}

The most common precipitant of DKA in adults is infection, which vary from gastrointestinal upset, with diarrhoea and vomiting, to chest or urinary tract infections. These precipitating illnesses need to be treated at the same time as the DKA. In addition, non-infectious illnesses, such as acute coronary syndrome that precipitate DKA need to be evaluated and addressed at the time of presentation. In children, episodes of DKA generally occur at onset or time of diagnosis of diabetes or because of insulin omission. Serious intercurrent illnesses are rarely present and routine investigation for precipitating causes of DKA is unnecessary.

\section{[H1] Quality of Life}

The UK National Institute for Health and Care Excellence (NICE) systematically reviewed the evidence for the management of DKA and found no studies in adults that evaluated quality of life ${ }^{204}$. However, fear of DKA is one of the factors affecting the quality of life in those with $\mathrm{T} 1 \mathrm{DM}^{205}$. Of note, despite the lower quality of life experienced by those with T1DM, recurrent DKA does not contribute to further reductions ${ }^{42}$. The development of any systemic or neurological injury can also lead to a reduction in quality of life and prevention of these complications remains a priority ${ }^{206}$. As mentioned previously, DKA remains an expensive condition to treat ${ }^{5-7}$. These costs place huge burdens on those who have to pay themselves and on society in general.

\section{[H2] Other complications}

DKA is associated with a wide range of complications. For example, hypokalaemia and hypoglycaemia are the most frequent complications of DKA treatment, but are generally mild and easily treated with ongoing careful biochemical monitoring ${ }^{22,35}$. Other important complications of DKA include the development of a hypercoagulable state with increased risk of deep venous thromboses, particularly when central venous catheters are used to gain intravenous access if peripheral access was not possible due to severe dehyration ${ }^{207}$. DKA also frequently causes acute kidney injury (AKI) in children. In one study, $64 \%$ of children with DKA 
were found to have $\mathrm{AKI} ;>50 \%$ had stage 2 or stage $3 \mathrm{AKI}$, suggesting renal tubular injury, rather than simply pre-renal uraemia due to circulatory volume depletion with renal hypoperfusion ${ }^{208}$. Other complications of DKA are rare (Table 3).

Patients with DKA with chronic poor glycaemic control are uniquely susceptible to rhinocerebral or pulmonary mucormycosis ${ }^{209}$, which is frequently fatal. Acidotic conditions decrease iron binding to transferrin, creating conditions that support fungal growth. Some rare complications of DKA include cardiac arrhythmias due to electrolyte derangements, intestinal necrosis, pulmonary oedema and pneumomediastinum (abnormal presence of air in the mediastinum), which might be associated with pneumothorax and is thought to be caused by protracted vomiting and hyperventilation 210,211 . Multiple organ dysfunction syndrome is another rare complication of DKA causing multiple organ failure, which may be associated with thrombocytopenia in children; reported cases in adults often involve elevated liver enzymes, elevated pancreatic enzymes and renal dysfunction ${ }^{212-214}$. Peripheral neuropathy has been reported in children, and might occur in association with other DKA complications including cerebral injury or disseminated intravascular coagulation ${ }^{215-219}$. Other isolated case reports have described rare neurological complications including cerebellar ataxia, movement disorder (choreiform movements and pill rolling tremor) and hemiparesis in children ${ }^{219}$.

\section{[H1] Outlook}

Increasing numbers of DKA hospitalizations highlight the need for targeted programmes to prevent DKA at new-onset of diabetes and recurrent episodes of DKA in children and adults with previously diagnosed diabetes. Education and the implementation of protocols aimed at maintenance insulin administration after discharge might reduce lapses in treatment and are a cost-effective way to reduce future risk of hospitalization for hyperglycaemic emergencies ${ }^{220}$. Several strategies including early screening, close follow-up of high-risk individuals (for example, those with multiple admissions), availability of telephone support from diabetes specialist nurses, and education of parents and communities have been proposed ${ }^{13,144}$. Studies have reported a lower incidence of DKA when parents were made aware of the higher risk of diabetes in their children (due to the presence of autoantibodies) ${ }^{221}$. Similarly, another study showed close follow-up of high-risk children in the prediabetes stage reduced hospitalizations for DKA ${ }^{222}$. In Italy, a prevention programme educating parents, paediatricians and school staff reduced the number of children presenting with DKA at initial diagnosis of diabetes ${ }^{223}$. In 1991 , when the study started, this programme cost $\$ 23,470$ to deliver, and led to a reduction of DKA 
as the presenting feature of diabetes from $78 \%$ to $12.5 \%$ over the 8 years of follow up. Thus, delivering targeted education to those who have most contact with children might be beneficial.

\section{[H2] Clinical priorities}

More intensive coordination of care with patients and greater family engagement are some of the additional strategies for prevention of recurrent episodes of DKA. The Novel Interventions in Children's Healthcare programme uses care coordination with family and telemedicine in an attempt as a part of the preventive strategy to engage young people with multiple hospitalizations for DKA ${ }^{224}$. This work used text messages and other forms of communication with the adolescents and showed that daily communication decreased DKA readmissions. Furthermore, the Type 1 Diabetes Exchange programme showed that the use of new technology such as insulin pumps and real-time continuous glucose monitoring could be useful in preventing recurrent $\mathrm{DKA}^{225-227}$.

In the 1990s, the use of CSII or insulin pumps was associated with increased risk of DKA in children and adults with T1DM ${ }^{228}$. A series from 2017, however, reported a low incidence of 1.0 case/100 patient years ${ }^{229}$. An analysis of 13,487 participants (aged 2-26 years) in the T1DM Exchange clinic registry found that a lower incidence of DKA in those treated with CSII than in patients treated with multi-dose subcutaneous insulin injections ${ }^{230}$. However, as these individuals were looked after in specialist diabetes centres in the USA, rates of DKA amongst those cared for in other centres may be higher. Similarly, in a German study in children with T1DM, those who used CSII had lower rates of DKA than those receiving insulin by injection (2.29 versus 2.80 per 100 patient-years) ${ }^{231}$, suggesting that increasing CSII use might be an alternative method for reducing DKA incidence. However, pump use is expensive and requires access to specialist centres with appropriate expertise.

Patients with treatment adherence problems account for a disproportionate number of recurrent DKA episodes. In the USA, 50\% of first episodes of DKA in adults with T2DM and $~ 80 \%$ of recurrent DKA episodes are caused by poor compliance with therapy ${ }^{42}$. In the UK, adults who had attended a structured diabetes education programme and were on a flexible basal-bolus insulin dosing regimen based on individualizing carbohydrate ratios at each meal experienced a $61 \%$ reduction in risk for $\mathrm{DKA}^{232}$. Similarly, a multidisciplinary, multi-pronged approach incorporating more flexible intensive insulin regimens, standardizing diabetes education and 
empowering community engagement, reported a 44\% reduction in DKA admissions in those with $\mathrm{T}_{1} \mathrm{DM}^{233}$. Future strategies to increase treatment adherence combining increased education, motivational interviews, patient support technology (continuous glucose monitoring, CSII, telephone support, text and e-mail messaging) are needed to improve adherence to therapy and to reduce the risk of DKA.

In less developed parts of the world, efforts need to be made to ensure easy availability of insulin at an affordable price. Insulin and $0.9 \%$ saline solution are on the WHO list of essential medicines $^{234}$. Education of local health care providers also remains key to the recognition of DKA as well as prompt access to health care facilities with the ability to administer appropriate care.

\section{[H2] Unmet needs and areas for future research}

To date, many of the guidelines used to treat DKA have evolved over time, which are largely based on consensus and opinion. Thus, large RCTs are needed to help determine the best management options including optimizing electrolyte content of intravenous fluids (for example, Ringer's lactate versus $0.9 \%$ saline $)^{151,152,235}$. In addition, further investigations are necessary to determine the optimal rates and optimal technique of insulin administration ${ }^{236}$. Additional studies are also needed to determine the ideal combination of potassium salts for replacement. In essence, most stages of the patient journey from the time of diagnosis and admission to the time of discharge has areas of uncertainty that need good quality data to help improve overall patient management. Furthermore, the advent of closed loop systems for those with T1DM where the subcutaneously implanted interstitial glucose sensor is wirelessly linked to an insulin pump and other 'artificial intelligence' systems may also improve outcomes. They have been shown to improve time in glucose range, and thus, the likelihood of developing hyperglycaemia and subsequent DKA may be reduced ${ }^{237,238}$. However, this has yet to be determined 
Table 1: Diagnostic Criteria for DKA.

973

\begin{tabular}{|c|c|c|c|c|c|c|c|c|}
\hline Severity & $\begin{array}{l}\text { Glucose } \\
(\mathrm{mg} / \mathrm{dl}) \\
(\mathrm{mmol} / \mathrm{L})\end{array}$ & $\begin{array}{l}\text { Arterial or } \\
\text { venous } \mathrm{pH}\end{array}$ & $\begin{array}{l}\text { Bicarbonate } \\
(\mathrm{mmol} / \mathrm{L})\end{array}$ & $\begin{array}{l}\text { Urine or serum } \\
\text { ketones } \\
\text { (nitroprusside } \\
\text { test) }\end{array}$ & $\begin{array}{l}\beta \text {-hydroxy } \\
\text { butyrate } \\
(\mathrm{mmol} / \mathrm{L})\end{array}$ & $\begin{array}{l}\text { Anion gap } \\
\text { (mmol/L) }\end{array}$ & Mental status & Refs \\
\hline \multicolumn{9}{|c|}{ American Diabetes Association criteria for adults } \\
\hline Mild & $\begin{array}{l}>250 \\
(13.8)\end{array}$ & 7.25-7.30 & $15-18$ & Positive & $>3.0$ & $>10$ & Alert & \multirow{3}{*}{8} \\
\hline Moderate & $\begin{array}{l}>250 \\
(13.8)\end{array}$ & 7.24-7.0 & $10-15$ & Positive & $>3.0$ & $>12$ & Alert/drowsy & \\
\hline Severe & $\begin{array}{l}>250 \\
(13.8)\end{array}$ & $<7.0$ & $<10$ & Positive & $>3.0$ & $>12$ & Stupor/coma & \\
\hline \multicolumn{9}{|c|}{ Joint British Diabetes Societies } \\
\hline NA & $\begin{array}{l}>200 \\
(11.1)\end{array}$ & $<7.30^{\mathrm{a}}$ & $<15$ & Positive & $>3.0$ & NA & NA & 130 \\
\hline \multicolumn{9}{|c|}{ International Society of Pediatric and Adolescent Diabetes } \\
\hline Mild & $\begin{array}{l}>200 \\
(11.1)\end{array}$ & $<7.30^{\mathrm{a}}$ & $<15$ & Positive & $>3.0$ & NA & NA & \multirow{3}{*}{22} \\
\hline Moderate & $\begin{array}{l}>200 \\
(11.1)\end{array}$ & $<7.2^{\mathrm{a}}$ & $<10$ & Positive & $>3.0$ & NA & NA & \\
\hline Severe & $\begin{array}{l}>200 \\
(11.1)\end{array}$ & $<7.1^{\mathrm{a}}$ & $<5$ & Positive & $>3.0$ & NA & NA & \\
\hline
\end{tabular}

Adapted from Refs ${ }^{8,22,130}$. The ADA criteria recommends the use of arterial $\mathrm{pH}$ be for diagnosis and venous $\mathrm{pH}$ as a guide to evaluate the need for bicarbonate therapy and to measure resolution. Note that severity of DKA is defined by the degree of acidosis and level of consciousness, not by the degree of hyperglycaemia or ketonaemia. NA, not applicable. avenous $\mathrm{pH}$ can be used to diagnose DKA. 
Table 2: Precipitating causes of diabetic ketoacidosis in adults by region.

\begin{tabular}{|l|l|l|l|l|l|}
\hline Region & $\begin{array}{c}\text { New-onset } \\
\text { diabetes mellitus } \\
\text { (\%) }\end{array}$ & \multicolumn{1}{|c|}{ Infection (\%) } & \multicolumn{1}{|c|}{$\begin{array}{c}\text { Poor treatment } \\
\text { adherence (\%) }\end{array}$} & Other (\%) & $\begin{array}{c}\text { Unknown } \\
\text { (\%) }\end{array}$ \\
\hline Australia & 5.7 & 28.6 & 40 & 25.7 & NR \\
\hline Brazil & 12.2 & 25 & 39 & 15 & 8.8 \\
\hline China & NR & 39.2 & 24 & 10.9 & 25.9 \\
\hline Indonesia & 3.3 & 58.3 & 13.3 & 17.1 & 8 \\
\hline South Korea & NR & 25.3 & 32.7 & 11.2 & 30.8 \\
\hline Nigeria & NR & 32.5 & 27.5 & 4.8 & 34.6 \\
\hline Spain & 12.8 & 33.2 & 30.7 & 23.3 & NR \\
\hline Syria & NR & 47.8 & 23.5 & 7.8 & 20.9 \\
\hline Taiwan & 18.2 & 31.7 & 27.7 & 6.2 & 16.2 \\
\hline UK & 6.1 & 44.6 & 19.7 & 10.9 & 18.7 \\
\hline USA & $17.2-23.8$ & $14.0-16.0$ & $41.0-59.6$ & $9.7-18.0$ & $3.0-4.2$ \\
\hline
\end{tabular}

Adapted from ${ }^{1,35}$. NR, not reported. Other causes include the use of medications that affect carbohydrate metabolism, insulin pump failure, or alcohol or drug misuse. 


\begin{tabular}{|c|c|c|c|c|}
\hline Complication & Frequency & Description & Risk factors & Refs \\
\hline Cerebral injury & $\begin{array}{l}0-3-0.9 \% \text { of } \\
\text { children, rare } \\
\text { in adults }\end{array}$ & $\begin{array}{l}\text { Cerebral oedema; cerebral } \\
\text { thromboses, haemorrhage } \\
\text { and infarction; posterior } \\
\text { reversible encephalopathy } \\
\text { syndrome has also been } \\
\text { described }\end{array}$ & $\begin{array}{l}\text { Impaired renal function, low } \\
\mathrm{pH} \text {, low } \mathrm{pCO}_{2} \text {, lack of rise in } \\
\text { measured serum } \mathrm{Na}^{+} \text {during } \\
\text { DKA treatment, low } \mathrm{Na}^{+} \text {at } \\
\text { presentation, high } \mathrm{K}^{+} \text {at } \\
\text { presentation }\end{array}$ & $186,195,203,239-241$ \\
\hline Acute kidney injury & $\begin{array}{l}30-64 \% \text { of } \\
\text { children, } \\
50 \% \text { of } \\
\text { adults }\end{array}$ & $\begin{array}{l}\text { Stage } 1 \text { (pre-renal) is most } \\
\text { common but stage } 2 \text { and } \\
\text { stage } 3 \text { occur in substantial } \\
\text { numbers of patients } \\
\text { (children); rare episodes of } \\
\text { renal failure; some episodes } \\
\text { of renal failure associated } \\
\text { with rhabdomyolysis (adults } \\
\text { and children) }\end{array}$ & $\begin{array}{l}\text { High acidaemia (children), high } \\
\text { heart rate (children), high } \\
\text { corrected } \mathrm{Na}^{+} \text {concentration } \\
\text { (children), older age, high } \\
\text { glucose (adults), low serum } \\
\text { protein (adults) }\end{array}$ & $208,242,243$ \\
\hline $\begin{array}{l}\text { Large vessel } \\
\text { thromboses }\end{array}$ & $\begin{array}{l}50 \% \text { of } \\
\text { children }^{\mathrm{b}}\end{array}$ & $\begin{array}{l}\text { Rare reports in children of } \\
\text { stroke and other } \\
\text { thromboses not associated } \\
\text { with central venous catheter } \\
\text { use. Thrombophilia in some } \\
\text { cases in children; fatal } \\
\text { pulmonary } \\
\text { thromboembolism as well } \\
\text { as thromboses in other } \\
\text { regions in adults }\end{array}$ & $\begin{array}{l}\text { Central venous catheter use, } \\
\text { DKA causes a } \\
\text { hypercoagulable state }\end{array}$ & 244-247 \\
\hline $\begin{array}{l}\text { Subclinical interstitial } \\
\text { pulmonary oedema }\end{array}$ & $\begin{array}{l}\text { Common in } \\
\text { children }^{\mathrm{b}}\end{array}$ & \multirow{2}{*}{$\begin{array}{l}\text { Generally subclinical but } \\
\text { rare episodes of ARDS } \\
\text { have been described; } \\
\text { episodes of simultaneous } \\
\text { pulmonary oedema and } \\
\text { cerebral oedema are } \\
\text { described in both adults and } \\
\text { children }\end{array}$} & \multirow{2}{*}{$\begin{array}{l}\text { Hypokalaemia or } \\
\text { hypophosphataemia in some } \\
\text { cases in adults and children }\end{array}$} & \multirow[t]{2}{*}{248,249} \\
\hline $\begin{array}{l}\text { Symptomatic } \\
\text { pulmonary oedema }\end{array}$ & $\begin{array}{l}\text { Rare in } \\
\text { adults and } \\
\text { children }\end{array}$ & & & \\
\hline $\begin{array}{l}\text { Pancreatic enzyme } \\
\text { elevation }\end{array}$ & $\begin{array}{l}20-30 \% \text { of } \\
\text { children, } 16- \\
29 \% \text { of } \\
\text { adults }\end{array}$ & \multirow{2}{*}{$\begin{array}{l}\text { Acute pancreatitis, } \\
\text { sometimes associated with } \\
\text { hypertriglyceridaemia or } \\
\text { alcohol; asymptomatic } \\
\text { pancreatic enzyme } \\
\text { elevation without acute } \\
\text { pancreatitis is common in } \\
\text { both children and adults; } \\
\text { pancreatitis is rare in } \\
\text { children }\end{array}$} & \multirow{2}{*}{$\begin{array}{l}\text { High acidaemia, impaired renal } \\
\text { function, hypophosphataemia } \\
\text { in adults and children }\end{array}$} & \multirow{2}{*}{$250-252$} \\
\hline Pancreatitis & $\begin{array}{l}2 \% \text { of } \\
\text { children, } 10- \\
11 \% \text { of } \\
\text { adults }\end{array}$ & & & \\
\hline Cardiac arrhythmias & $\begin{array}{l}47 \% \text { of } \\
\text { children }^{\mathrm{b}}\end{array}$ & $\begin{array}{l}\text { Prolonged QTc occurs } \\
\text { commonly but is } \\
\text { asymptomatic; Brugada } \\
\text { pattern of arrhythmia has } \\
\text { been described in multiple } \\
\text { adult and paediatric case } \\
\text { reports; Electrolyte } \\
\text { abnormalities including } \\
\text { hypophosphataemia has } \\
\text { been shown to cause rare } \\
\text { episodes of arrhythmia }\end{array}$ & $\begin{array}{l}\text { In adults and children high } \\
\text { anion gap (QTc), } \\
\text { hypokalaemia, } \\
\text { hypophosphataemia and } \\
\text { hyperkalaemia }\end{array}$ & $253-258$ \\
\hline
\end{tabular}




\begin{tabular}{|c|c|c|c|c|}
\hline $\begin{array}{l}\text { Subtle or asymptomatic } \\
\text { diastolic dysfunction }\end{array}$ & $\begin{array}{l}47 \% \text { of } \\
\text { children }^{b}\end{array}$ & \multirow{2}{*}{$\begin{array}{l}\text { Asymptomatic elevations of } \\
\text { cardiac troponin I and CK- } \\
\text { MB detected in children; } \\
\text { might be associated with } \\
\text { systemic inflammatory } \\
\text { response; possibly } \\
\text { associated with thiamine } \\
\text { deficiency }\end{array}$} & \multirow{2}{*}{$\begin{array}{l}\text { High acidaemia; presence of } \\
\text { the systemic inflammatory } \\
\text { response }\end{array}$} & \multirow[t]{2}{*}{$259-262$} \\
\hline $\begin{array}{l}\text { Symptomatic } \\
\text { cardiomyopathy }\end{array}$ & $\begin{array}{l}\text { Rare in } \\
\text { adults and } \\
\text { children }\end{array}$ & & & \\
\hline Rhabdomyolysis & $\begin{array}{l}16 \% \text { of } \\
\text { adults, } 10 \% \\
\text { of children }\end{array}$ & $\begin{array}{l}\text { Often subclinical; occurs } \\
\text { more frequently in HHS but } \\
\text { also described in DKA; } \\
\text { some cases are associated } \\
\text { with hypophosphataemia. } \\
\text { Severe rhabdomyolysis are } \\
\text { mainly described in mixed } \\
\text { DKA and HHS and in } \\
\text { severe hypophosphataemia }\end{array}$ & $\begin{array}{l}\text { Low } \mathrm{pH} \text {, impaired renal } \\
\text { function, High glucose and } \\
\mathrm{Na}^{+} \text {, hypophosphataemia, } \\
\text { increased osmolality }\end{array}$ & $191,263-266$ \\
\hline $\begin{array}{l}\text { Asymptomatic } \\
\text { hypophosphataemia }\end{array}$ & $\begin{array}{l}\text { Up to } 90 \% \text { of } \\
\text { adults }^{c}\end{array}$ & \multirow{2}{*}{$\begin{array}{l}\text { Asymptomatic } \\
\text { hypophosphataemia is } \\
\text { common; case reports of } \\
\text { severe hypophosphataemia } \\
\text { causing rhabdomyolysis, } \\
\text { renal failure, haemolytic } \\
\text { anaemia, arrhythmia, } \\
\text { respiratory failure }\end{array}$} & \multirow[t]{2}{*}{ High acidaemia } & \multirow[t]{2}{*}{$98,188-191$} \\
\hline $\begin{array}{l}\text { Severe or } \\
\text { symptomatic } \\
\text { hypophosphataemia }\end{array}$ & $\begin{array}{l}\text { Rare in } \\
\text { adults and } \\
\text { children }\end{array}$ & & & \\
\hline $\begin{array}{l}\text { Intestinal necrosis or } \\
\text { GI bleeding }\end{array}$ & $\begin{array}{l}\text { Rare in } \\
\text { children, } \\
\text { upper Gl } \\
\text { bleeding in } \\
9 \% \text { of adults }\end{array}$ & $\begin{array}{l}\text { Intestinal necrosis thought } \\
\text { to be related to } \\
\text { hypoperfusion and } \\
\text { microangiopathy; intestinal } \\
\text { necrosis is described in } \\
\text { children and adolescents } \\
\text { but not adults, upper Gl } \\
\text { bleeding is frequent in } \\
\text { adults, which might be } \\
\text { related to acid reflux during } \\
\text { DKA }\end{array}$ & $\begin{array}{l}\text { Impaired renal function, high } \\
\text { glucose }\end{array}$ & 267,268 \\
\hline
\end{tabular}

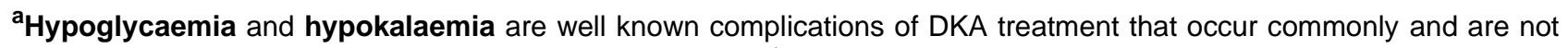
included here as they are discussed extensively in the text. ${ }^{b}$ Rates in adults are unknown; ${ }^{\circ}$ Rates in children unknown ARDS, acute respiratory distress syndrome; CK-MB, creatine kinase - myocardial band; DKA, diabetic ketoacidosis; GI, gastrointestinal; $\mathrm{HHS}$, hyperglycaemic hyperosmolar state, $\mathrm{pCO}_{2}$, partial pressure of carbon dioxide; QTc, corrected QT interval. 


\section{Figure legends}

\section{Figure 1. The history of DKA.}

The first reports of diabetic coma date back to the early 1800s and included isolated cases of children and adults with previously undiagnosed or established diabetes who presented with rapid onset symptoms of hyperglycaemia that led to coma and death ${ }^{269}$. In 1857 , the presence of acetone was identified in the urine of an individual presenting in a diabetic coma ${ }^{270}$. Two decades later, the German physician Adolf Kussmaul reported severe dyspnoea (hyperventilation) in patients ${ }^{271}$. A decade later, Stadelmann reported that the urine of most patients with diabetic coma contained large quantities of $\beta$-hydroxybutyric acid, in addition to acetoacetate ${ }^{272}$. The mortality rate was $>90 \%$ in the pre-insulin era ${ }^{273}$ with only a few patients living longer than a few months. In subsequent decades, the mortality associated with DKA decreased to $<1-2 \%$ since the 2010 s in developed countries $^{1,8}$. It was not until in the 1970 s that it was established that low-dose intravenous insulin infusions were introduced following data to show that they lowered glucose and ketone concentrations just as well as higher doses ${ }^{274}$. The first American Diabetes Association (ADA) guideline was published in 2001. and the first edition of the UK guideline was published in 2011. In 2018, the first randomized controlled trial of fluid replacement in children showed no differences in acute or post-recovery neurological outcomes in children with DKA treated with rapid versus slower volume correction using either $0.9 \%$ or $0.45 \%$ saline $e^{159}$.

\section{Figure 2: Pathogenesis of diabetic ketoacidosis.}

Hyperglycaemia develops in insulin deficiency because of three processes: increased gluconeogenesis, accelerated glycogenolysis, and impaired glucose utilization by peripheral tissues. The reduction in insulin concentration together with the increase in counter-regulatory hormones, leads to the activation of hormone sensitive lipase in adipose tissue with the subsequent breakdown of triglyceride into glycerol and free fatty acids (FFAs). In the liver, FFAs are oxidized to ketoacids, mainly under the influence of glucagon. FFAs undergo $\beta$-oxidation to form acetyl CoA. Excess acetyl CoA that does not enter the Krebs cycle generates acetoacetyl CoA, three molecules of which condense to form hydroxyl-3-methylgluterate-CoA (HMG-CoA). This is turn is cleaved to form acetoacetate and acetyl CoA. The acetoacetate is further reduced by $\mathrm{NADH}$ to form $\beta$-hydroxybutyrate. The two major ketoacids are $\beta$-hydroxybutyrate and acetoacetate. Accumulation of ketoacids leads to a high anion gap metabolic acidosis due to the 
reduction in serum bicarbonate concentration and 'fixed acid' retention. Hyperglycaemia also activates macrophages to produce pro-inflammatory cytokines, and the liver to produce CRP, which in turn impair pancreatic $\beta$-cell function, as well as reducing endothelial nitric oxide, leading to endothelial dysfunction. Hyperglycaemia and high ketone levels cause an osmotic diuresis that leads to hypovolaemia, decreased glomerular filtration rate worsening hyperglycaemia. As a result of respiratory compensation for the metabolic acidosis, Kussmaul breathing characterized by deep, regular breaths (often with a 'fruity' odour) are taken by those in DKA as a way of excreting acidic carbon dioxide. Cerebral oedema is increased fluid content of the brain tissue that may lead to neurological signs and symptoms.

\section{Figure 3: Symptoms and signs of DKA.}

The osmotic diuresis of hyperglycaemia and ketonuria causes circulatory volume depletion. This in turn can cause the lethargy, stupor and coma. The metabolic acidosis stimulates respiratory compensation, with the classic hyperventilation ('air hunger') that is Kussmaul breathing - the volatile ketones can be smelt on the breath. Changes in visual acuity, which is thought to be due to changes in water content in the eye ball or the lens are also observed. Patients with diabetic ketoacidosis also experience abdominal pain, nausea and vomiting that resolve with treatment. 


\section{Box 1. Hyperglycaemic hyperosmolar state}

Hyperglycaemic hyperosmolar state (HHS) is another commonly encountered hyperglycaemic emergency. HHS occurs less frequently than DKA ( $<1 \%$ of diabetes-related emergencies ${ }^{269}$ ), but has a substantial mortality of up to $20 \%{ }^{149,269}$. HHS is characterized by severe hyperglycaemia and high serum osmolality (concentration of electrolytes and glucose in the serum) accompanied by circulatory volume depletion ${ }^{275}$. In HHS, insulin concentrations are adequate to inhibit ketogenesis, but not high enough to ensure adequate cellular glucose uptake. So, HHS is characterized by hyperglycaemia and an osmotic diuresis that perpetuates dehydration without ketosis. As with DKA, concurrent illness, such as infection or acute coronary syndrome can lead to an increase in counter-regulatory hormones, which exacerbates hyperglycaemia. Medications such as corticosteroids and atypical antipsychotics can also precipitate $\mathrm{HHS}^{276,277}$.

The UK and US guidelines for diagnosing HHS slightly differ from eachother ${ }^{8,275}$. The UK guidelines define HHS as a glucose concentration $\geq 30 \mathrm{mmol} / \mathrm{l}, \mathrm{pH}>7.3$, bicarbonate $>15 \mathrm{mmol} / \mathrm{l}$, and blood $\beta$-hydroxybutyrate $<3.0 \mathrm{mmol} / \mathrm{l}$, and osmolality of $>320 \mathrm{mosmol} /{ }^{275}$; US guidelines define HHS as glucose levels $>33.3 \mathrm{mmol} / \mathrm{l}, \mathrm{pH}>7.3$, bicarbonate $>18 \mathrm{mmol} / \mathrm{l}$, with 'small' concentrations or urinary or serum ketones and osmolality of $>320 \mathrm{mosmol} / /^{8}$. In addition to detecting and treating any precipitating cause, the management of $\mathrm{HHS}$ involves correction of fluid deficits including potassium replacement and reducing hyperosmolality. The administration of intravenous fluids, such as $0.9 \%$ saline will also lower glucose concentrations by addressing the haemoconcentration (an increase in the proportion of the blood that is cells, due to the loss of water) and restoring renal perfusion. Circulatory volume depletion is more severe in HHS than in DKA and higher rates of fluid administration are typically necessary. Consensus recommendations from various groups are slightly different owing to lack of trials ${ }^{8,275}$. Intravenous insulin is started immediately after the initial fluid bolus if there is evidence of a metabolic acidosis (DKA and HHS can frequently co-exist ${ }^{278}$ ). However, in the absence of acidosis, a weight-based fixed rate intravenous insulin infusion is started only after the glucose concentration ceases to decline with fluid replacement alone ${ }^{275}$, or after potassium levels have been corrected $^{8}$. 


\section{Box 2. Current potassium replacement guidelines}

\section{[H1] Adults}

- $\mathrm{K}^{+} \geq 5.5 \mathrm{mmol} / \mathrm{l}: \mathrm{no}$ supplementation is required due to the risk of precipitating cardiac arrhythmias with additional potassium

- $\mathrm{K}^{+}=4.0-5.0 \mathrm{mmol} / \mathrm{l}: 20 \mathrm{mmol} / \mathrm{l}$ of replacement fluid

- $\mathrm{K}^{+}=3.0-4.0 \mathrm{mmol} / \mathrm{l}: 40 \mathrm{mmol} / \mathrm{l}$ of replacement fluid

- $\mathrm{K}^{+}=<3.0 \mathrm{mmol} / \mathrm{l}: 10-20 \mathrm{mmol}$ per hour until serum $\mathrm{K}^{+}>3.0 \mathrm{mmol} / \mathrm{l}$, then add $40 \mathrm{mmol} / \mathrm{l}$ to replacement fluid.

\section{[H1] Children}

- $\mathrm{K}^{+}>5.0 \mathrm{mmol} / \mathrm{l}$ : delay potassium administration until $\mathrm{K}^{+} \leq 5.0 \mathrm{mmol} / \mathrm{l}$.

- $\mathrm{K}^{+} 3.5-5.0 \mathrm{mmol} / \mathrm{l}$ : add potassium $40 \mathrm{mmol} / \mathrm{l}$ to the infusion after administering the initial fluid replacement bolus.

- $\mathrm{K}^{+}<3.5 \mathrm{mmol} / \mathrm{l}$ : begin potassium replacement $40 \mathrm{mmol} / \mathrm{l}$ as soon as possible and delay insulin administration until potassium level is normal. 


\section{Glossary terms}

\section{BMI z-score}

Also known as the BMI standard deviation scores, the z-score is a measure of a child's relative weight adjusted for age and gender

\section{Buffering}

The ability of molecules in the circulation to stabilise the acid base balance in an attempt to maintain the $\mathrm{pH}$

\section{pKa}

This is the negative base-10 logarithm of the acid dissociation constant $(\mathrm{Ka})$ of a solution. The lower the $\mathrm{pKa}$, the stronger the acid.

\section{Circulatory volume depletion}

A reduction in intravascular and / or extracellular fluid volume, such that there may be an inability to adequately perfuse tissue.

\section{Glomerular filtration rate}

This is an estimate of how much blood passes through the renal glomeruli every minute. Is it often a calculation from the serum creatinine, age, gender and body weight

Hypertonicity - A state where the circulating extracellular fluid has a higher osmotic pressure, than would be observed in a healthy individual.

\section{Pre-renal renal failure}

The loss of kidney function as a result of poor renal or glomerular perfusion, e.g. haemorrhage, cardiac failure or hypovolaemia. 
8. Kitabchi,A.E., Umpierrez,G.E., Miles,J.M. \& Fisher,J.N. Hyperglycemic crises in adult patients with diabetes. Diabetes Care 32, 1335-1343 (2009)

These - current - guidelines from the American Diabetes Association remain amongst the most used in the world for adults.

10. Benoit,S.R., Zhang,Y., Geiss,L.S., Gregg,E.W. \& Albright,A. Trends in diabetic ketoacidosis hospitalizations and in-hospital mortality - United States, 2000-2014. Morb. Mortal. Wkly. Rep. 67, 362-365 (2018)

This report from the Centers for Disease Control illustrates the trends in DKA in the USA over the first 15 years of this Century

21. Rewers,A. et al. Presence of diabetic ketoacidosis at diagnosis of diabetes mellitus in youth: The Search for Diabetes in Youth Study. Pediatrics 121, e1258 (2008)

This large dataset showed that in the USA, 1 in 4 people under the age of 20 years prsented with DKA when first diagnosed with diabetes.

22. Wolfsdorf,J.I. et al. ISPAD clinical practice consensus guidelines 2018: Diabetic ketoacidosis and the hyperglycemic hyperosmolar state. Pediatr. Diabetes 19, S155-S177 (2018)

These - current - guidelines from the International Society for Pediatric and Adolescent Diabetes remain amongst the most used in the world for children.

47. Maahs,D.M. et al. Rates of diabetic ketoacidosis: International comparison with 49,859 pediatric patients with type 1 diabetes from England, Wales, the U.S., Austria, and Germany. Diabetes Care 38, 1876-1882 (2015)

This very large data set looks at the the risk factors for developing DKA in children under 18 years with established T1DM

35. Dhatariya,K.K., Nunney,I., Higgins,K., Sampson,M.J. \& Iceton,G. A national survey of the management of diabetic ketoacidosis in the UK in 2014. Diabetic Med. 33, 252-260 (2016)

This work shows that the UK guideline works but that hypoglycaemia and hypokalaemia were common

65. Fadini,G.P., Bonora,B.M. \& Avogaro,A. SGLT2 inhibitors and diabetic ketoacidosis: data from the FDA Adverse Event Reporting System. Diabetologia 60, 1385-1389 (2017)

This highlights the relationship between SGLT2 inhibitor use and the risk of developing DKA

130. Savage,M.W. et al. Joint British Diabetes Societies guideline for the management of diabetic ketoacidosis. Diabetic Med. 28, 508-515 (2011)

The UK guideline on the management of DKA is also widely used across the world

159. Kuppermann,N. et al. Clinical trial of fluid infusion rates for pediatric diabetic ketoacidosis. N. Eng. J. Med. 378, 2275-2287 
This large trial showed that neither the rate of fluid replacement or the type of fluid used was associated with adverse neurological outcomes in children under 18 years

160. Grimberg,A., Cerri,R.W., Satin-Smith,M. \& Cohen,P. The "two bag system" for variable intravenous dextrose and fluid administration: Benefits in diabetic ketoacidosis management. J. Pediatr 134, 376-378 (1999

This retrospective analysis showed that in children the 'two bag' system was more cost effective and improved quality of care than a 'one bag' system.

168. Nallasamy,K., Jayashree,M., Singhi,S. \& Bansal,A. Low-dose vs standard-dose insulin in pediatric diabetic ketoacidosis: A randomized clinical trial. JAMA Pediatrics 168, 999-1005 (2014).

This trial showed that in children aged $\leq 12$ years, the rates of glucose decline and resolution of acidosis was the same when comparing insulin given at $0.05 \mathrm{units} / \mathrm{Kg} / \mathrm{hr}$ to 0.1 units $/ \mathrm{Kg} / \mathrm{hr}$

186. Glaser,N. et al. Risk factors for cerebral edema in children with diabetic ketoacidosis. N. Eng. J. Med. 344, 264-269 (2001)

This large dataset suggested that the key risk factors for developing cerebral oedema in children presenting with DKA were a low arterial $\mathrm{pCO}_{2}$, and high urea at presentation

208. Hursh,B.E., Ronsley,R., Islam,N., Mammen,C. \& Panagiotopoulos,C. Acute kidney injury in children with type 1 diabetes hospitalized for diabetic ketoacidosis. JAMA Pediatrics 171, e170020 (2017).

These data showed that acute kidney injury is seen in almost $65 \%$ in children presenting with DKA, with severity of DKA being a significant risk factor. 
1. Umpierrez,G. \& Korytkowski,M. Diabetic emergencies - ketoacidosis, hyperglycaemic hyperosmolar state and hypoglycaemia. Nat. Rev. Endocrinol. 12, 222-232 (2016).

2. Umpierrez,G.E., Smiley,D. \& Kitabchi,A.E. Narrative review: Ketosis-prone type 2 diabetes mellitus. Ann. Intern. Med. 144, 350-357 (2006).

3. Vellanki,P. \& Umpierrez,G.E. Diabetic ketoacidosis: A common debut of diabetes among African Americans with type 2 diabetes. Endocr. Pract. 23, 971-978 (2017).

4. Centers for Disease Control and Prevention. Age-adjusted hospital discharge rates for diabetic ketoacidosis as first-listed diagnosis per 10,000 population, United States, 1988-2009. http://www.cdc.gov/diabetes/statistics/dkafirst/fig7.htm. 2013. [Last accessed 20th March 2020]

5. Desai,D., Mehta,D., Mathias,P., Menon,G. \& Schubart,U.K. Health care utilization and burden of diabetic ketoacidosis in the U.S. over the past decade: A nationwide analysis. Diabetes Care 41, 1631-1638 (2018).

6. Dhatariya,K.K., Skedgel,C. \& Fordham,R. The cost of treating diabetic ketoacidosis in the UK: a national survey of hospital resource use. Diabetic Med. 34, 1361-1366 (2017).

7. Dhatariya,K.K. et al. The cost of treating diabetic ketoacidosis in an adolescent population in the UK: a national survey of hospital resource use. Diabetic Med. 36, 982-987 (2019).

8. Kitabchi,A.E., Umpierrez,G.E., Miles,J.M. \& Fisher,J.N. Hyperglycemic crises in adult patients with diabetes. Diabetes Care 32, 1335-1343 (2009).

9. Kitabchi,A.E. et al. Management of hyperglycemic crises in patients with diabetes. Diabetes Care 24, 131-153 (2001).

10. Benoit,S.R., Zhang,Y., Geiss,L.S., Gregg,E.W. \& Albright,A. Trends in diabetic ketoacidosis hospitalizations and in-hospital mortality - United States, 2000-2014. Morb. Mortal. Wkly. Rep. 67, 362-365 (2018).

11. Zhong,V.W., Juhaeri,J. \& Mayer-Davis,E.J. Trends in hospital admission for diabetic ketoacidosis in adults with type 1 and type 2 diabetes in England, 1998-2013: A retrospective cohort study. Diabetes Care 41, 1870-1877 (2018).

12. Poovazhagi,V. Risk factors for mortality in children with diabetic ketoacidosis from developing countries. World J. Diabetes 5, 932-938 (2014).

13. Vellanki,P. \& Umpierrez,G.E. Increasing hospitalizations for DKA: A need for prevention programs. Diabetes Care 41, 1839-1841 (2018). 
14. Dabelea,D. et al. Trends in the prevalence of ketoacidosis at diabetes diagnosis: The SEARCH for Diabetes in Youth Study. Pediatrics 133, e938-e945 (2014).

15. Neu,A. et al. Ketoacidosis at diabetes onset is still frequent in children and adolescents. Diabetes Care 32, 1647-1648 (2009).

16. Limenis,E., Shulman,R. \& Daneman,D. Is the frequency of ketoacidosis at onset of type 1 diabetes a child health indicator that is related to income inequality? Diabetes Care 35, e5 (2012).

17. Jefferies,C.A. et al. Preventing diabetic ketoacidosis. Pediatr. Clin. N. Am. 62, 857871 (2015).

18. Davis,A.K. et al. Prevalence of detectable C-peptide according to age at diagnosis and duration of type 1 diabetes. Diabetes Care 38, 476-481 (2015).

19. Usher-Smith,J.A., Thompson,M., Ercole,A. \& Walter,F.M. Variation between countries in the frequency of diabetic ketoacidosis at first presentation of type 1 diabetes in children: a systematic review. Diabetologia 55, 2878-2894 (2012).

20. Pinkney,J.H., Bingley,P.J., Sawtell,P.A., Dunger,D.B. \& Gale,E.A. Presentation and progress of childhood diabetes mellitus: a prospective population-based study. Diabetologia 37, 70-74 (1994).

21. Rewers,A. et al. Presence of diabetic ketoacidosis at diagnosis of diabetes mellitus in youth: The Search for Diabetes in Youth Study. Pediatrics 121, e1258 (2008).

22. Wolfsdorf,J.I. et al. ISPAD clinical practice consensus guidelines 2018: Diabetic ketoacidosis and the hyperglycemic hyperosmolar state. Pediatr. Diabetes 19, S155-S177 (2018).

23. Klingensmith,G.J. et al. Presentation of youth with type 2 diabetes in the Pediatric Diabetes Consortium. Pediatr. Diabetes 17, 266-273 (2016).

24. Mulukutla,S.N., Acevedo-Calado,M., Hampe,C.S., Pietropaolo,M. \& Balasubramanyam,A. Autoantibodies to the IA-2 extracellular domain refine the definition of "A+" subtypes of ketosis-prone diabetes. Diabetes Care 41, 2637-2640 (2018).

25. Farsani,S.F. et al. Incidence and prevalence of diabetic ketoacidosis (DKA) among adults with type 1 diabetes mellitus (T1D): a systematic literature review. BMJ Open 7, e016587 (2017).

26. Venkatesh,B. et al. Incidence and outcome of adults with diabetic ketoacidosis admitted to ICUs in Australia and New Zealand. Critical Care (London) 19, 451 (2016). 
27. Henriksen,O.M., Roder,M.E., Prahl,J.B. \& Svendsen,O.L. Diabetic ketoacidosis in Denmark. Diabetes Res. Clin. Pract. 76, 51-56 (2007).

28. Diaz-Valencia,P.A., Bougneres,P. \& Valleron,A.J. Global epidemiology of type 1 diabetes in young adults and adults: a systematic review. BMC Public Health 15, 255 (2015).

29. Li,J. et al. Secondary diabetic ketoacidosis and severe hypoglycaemia in patients with established type 1 diabetes mellitus in China: a multicentre registration study. Diabetes Metab. Res. Rev. 30, 497-504 (2014).

30. Liu,C.C. et al. Trends in hospitalization for diabetic ketoacidosis in diabetic patients in Taiwan: Analysis of national claims data, 1997-2005. J. Formos. Med. Assoc. 109, 725-734 (2010).

31. Lombardo,F., Maggini,M., Gruden,G. \& Bruno,G. Temporal trend in hospitalizations for acute diabetic complications: A nationwide study, Italy, 2001-2010. PLoS ONE 8, e63675 (2013).

32. Kalscheuer,H. et al. Event rates and risk factors for the development of diabetic ketoacidosis in adult patients with type 1 diabetes: Analysis from the DPV registry based on 46,966 patients. Diabetes Care 42, e34-e36 (2019).

33. Mays,J.A. et al. An evaluation of recurrent diabetic ketoacidosis, fragmentation of care, and mortality across Chicago, Illinois. Diabetes Care 39, 1671-1676 (2016).

34. Skinner,T.C. Recurrent diabetic ketoacidosis: Causes, prevention and management. Horm. Res. 57, 78-80 (2002).

35. Dhatariya,K.K., Nunney,I., Higgins,K., Sampson,M.J. \& Iceton,G. A national survey of the management of diabetic ketoacidosis in the UK in 2014. Diabetic Med. 33, 252-260 (2016).

36. Gibb,F.W., Teoh,W.L., Graham,J. \& Lockman,K.A. Risk of death following admission to a UK hospital with diabetic ketoacidosis. Diabetologia 59, 2082-2087 (2016).

37. Azevedo,L.C., Choi,H., Simmonds,K., Davidow,J. \& Bagshaw,S.M. Incidence and long-term outcomes of critically ill adult patients with moderate-to-severe diabetic ketoacidosis: Retrospective matched cohort study. J. Crit. Care 29, 971-977 (2014).

38. Große,J. et al. Incidence of diabetic ketoacidosis of new-onset type 1 diabetes in children and adolescents in different countries correlates with human development index (HDI): An updated systematic review, meta-analysis, and meta-regression. Horm. Metab. Res. 50, 209-222 (2018).

39. Agarwal,A. et al. Prognostic factors in patients hospitalized with diabetic ketoacidosis. Endocrinol. Metab 31, 424-432 (2016). 
40. Chung,S.T. et al. Predictors of hyperglycaemic crises and their associated mortality in Jamaica. Diabetes Res Clin Pract 73, 184-190 (2006).

41. Otieno,C.F., Kayima,J.K., Omonge,E.O. \& Oyoo,G.O. Diabetic ketoacidosis: risk factors, mechanisms and management strategies in sub-Saharan Africa: a review. E. Afr. Med. J 82, S197-203 (2005).

42. Randall,L. et al. Recurrent diabetic ketoacidosis in inner-city minority patients. Diabetes Care 34, 1891-1896 (2011).

43. Torjesen,I. Diabulimia: the world's most dangerous eating disorder. BMJ 364, 1982 (2019).

44. Lindner,L.M., Rathmann,W. \& Rosenbauer,J. Inequalities in glycaemic control, hypoglycaemia and diabetic ketoacidosis according to socio-economic status and area-level deprivation in Type 1 diabetes mellitus: a systematic review. Diabetic Med. 35, 12-32 (2017).

45. Foster,N.C. et al. State of type 1 diabetes management and outcomes from the T1D Exchange in 2016-2018. Diabetes Technol. Ther. 21, 66-72 (2019).

46. Shah,V.N. et al. Gender differences in diabetes self-care in adults with type 1 diabetes: Findings from the T1D Exchange clinic registry. J. Diabetes Complications 32, 961-965 (2018).

47. Maahs,D.M. et al. Rates of diabetic ketoacidosis: International comparison with 49,859 pediatric patients with type 1 diabetes from England, Wales, the U.S., Austria, and Germany. Diabetes Care 38, 1876-1882 (2015).

48. Hurtado,C.R. et al. Causes and predictors for 30-day re-admissions in adult patients with diabetic ketoacidosis in the United States: A nationwide analysis, 210-2014. Endocr. Pract. 25, 242-253 (2019).

49. Del Degan,S., Dube,F., Gagnon,C. \& Boulet,G. Risk factors of recurrent diabetic ketoacidosis in adults with type 1 diabetes. Can. J. Diabetes 43, 472-476.e1 (2019).

50. Bui,H., To,T., Stein,R., Fung,K. \& Daneman,D. Is diabetic ketoacidosis at disease onset a result of missed diagnosis? J. Pediatr 156, $472-477$ (2010).

51. Flood,R.G. \& Chiang,V.W. Rate and prediction of infection in children with diabetic ketoacidosis. Am. J. Emerg. Med. 19, 270-273 (2001).

52. Wolfsdorf,J.I. et al. Diabetic ketoacidosis and hyperglycemic hypersmolar state. Pediatr. Diabetes 15, 154-179 (2014).

53. Edge,J.A., Nunney,I. \& Dhatariya,K.K. Diabetic ketoacidosis in an adolescent and young adult population in the UK in 2014: a national survey comparison of management in paediatric and adult settings. Diabetic Med. 33, 1352-1359 (2016). 
54. Praveen,P.A. et al. Diabetic ketoacidosis at diagnosis among youth with type 1 and type 2 diabetes: Results from SEARCH (United States) and YDR (India) registries. Pediatr. Diabetes https://doi.org/10.1111/pedi.12979, (2020).

55. Pinhas-Hamiel,O., Hamiel,U. \& Levy-Shraga,Y. Eating disorders in adolescents with type 1 diabetes: Challenges in diagnosis and treatment. World Journal Diabetes 6 , 517-526 (2015).

56. Garrett,C.J., Choudhary,P., Amiel,S.A., Fonagy,P. \& Ismail,K. Recurrent diabetic ketoacidosis and a brief history of brittle diabetes research: contemporary and past evidence in diabetic ketoacidosis research including mortality, mental health and prevention. Diabetic Med. 36, 1329-1335 (2019).

57. Polonsky,W.H. et al. Insulin omission in women with IDDM. Diabetes Care 17, 1178-1185 (1994).

58. Rydall,A.C., Rodin,G.M., Olmsted,M.P., Devenyi,R.G. \& Daneman,D. Disordered eating behavior and microvascular complications in young women with insulindependent diabetes mellitus. N. Eng. J. Med. 336, 1849-1854 (1997).

59. Price,H.C., Ismail,K. \& on Behalf of the Joint British Diabetes Societies (JBDS) for Inpatient Care. Royal College of Psychiatrists Liaison Faculty \& Joint British Diabetes Societies (JBDS): guidelines for the management of diabetes in adults and children with psychiatric disorders in inpatient settings. Diabetic Med. 35, 9971004 (2018).

60. Healthcare Quality Improvement Partnership \& Royal College of Paediatrics and Child Health. National Paediatric Diabetes Audit Report 2012-15: Part 2. Hospital admissions and complications. https://www.rcpch.ac.uk/sites/default/files/201803/npda hospital admissions report part 2 2012-15.pdf. 2017. [Last accessed 20th March 2020]

61. Erondu,N., Desai,M., Ways,K. \& Meininger,G. Diabetic ketoacidosis and related events in the canagliflozin type 2 diabetes clinical program. Diabetes Care 38, 1680-1686 (2015).

62. Henry,R.R., Thakkar,P., Tong,C., Polidori,D. \& Alba,M. Efficacy and safety of canagliflozin, a sodium-glucose cotransporter 2 inhibitor, as add-on to insulin in patients with type 1 diabetes. Diabetes Care 38, 2258-2265 (2015).

63. Goldenberg,R.M. et al. SGLT2 inhibitor-associated diabetic ketoacidosis: Clinical review and recommendations for prevention and diagnosis. Clin. Ther. 38, 26542664.e1 (2016).

64. Matthaei,S., Bowering,K., Rohwedder,K., Grohl,A. \& Parikh,S. Dapagliflozin improves glycemic control and reduces body weight as add-on therapy to metformin plus sulfonylurea: A 24-week randomized, double-blind clinical trial. Diabetes Care 38, 365-372 (2015). 
65. Fadini,G.P., Bonora,B.M. \& Avogaro,A. SGLT2 inhibitors and diabetic ketoacidosis: data from the FDA Adverse Event Reporting System. Diabetologia 60, 1385-1389 (2017).

66. Danne,T. et al. International consensus on risk management of diabetic ketoacidosis in patients with type 1 diabetes treated with sodium-glucose cotransporter (SGLT) inhibitors. Diabetes Care 42, 1147-1154 (2019).

67. Garg,S.K., Peters,A.L., Buse,J.B. \& Danne,T. Strategy for mitigating DKA risk in patients with type 1 diabetes on adjunctive treatment with SGLT inhibitors: A STICH protocol. Diabetes Technol. Ther. 20, 571-575 (2018).

68. European Medicines Agency. First oral add-on treatment to insulin for treatment of certain patients with type 1 diabetes. https://www.ema.europa.eu/en/news/first-oraladd-treatment-insulin-treatment-certain-patients-type-1-diabetes. 2019. [Last accessed 20th March 2020]

69. www.Drugs.com. Pramlintide. https://www.drugs.com/ppa/pramlintide.html. 2019. [Last accessed 20th March 2020]

70. Kinney,G.L., Akturk,H.K., Taylor,D.D., Foster,N.C. \& Shah,V.N. Cannabis use is associated with increased risk for diabetic ketoacidosis in adults with type 1 diabetes: Findings from the T1D Exchange Clinic Registry. Diabetes Care 43, 247249 (2020).

71. Guenette,M.D., Hahn,M., Cohn,T.A., Teo,C. \& Remington,G.J. Atypical antipsychotics and diabetic ketoacidosis: a review. Psychopharmacology 226, 1-12 (2013).

72. Ananth,J., Parameswaran,S. \& Gunatilake,S. Side effects of atypical antipsychotic drugs. Curr. Pharm. Des. 10, 2219-2229 (2004).

73. Ribas,A. \& Wolchok,J.D. Cancer immunotherapy using checkpoint blockade. Science 359, 1350-1355 (2018).

74. Akturk,H.K. et al. Immune checkpoint inhibitor-induced Type 1 diabetes: a systematic review and meta-analysis. Diabetic Med. 36, 1075-1081 (2019).

75. Wright,J.J. et al. Increased reporting of immune checkpoint inhibitor-associated diabetes. Diabetes Care 41, e150-e151 (2018).

76. Stamatouli,A.M. et al. Collateral damage: Insulin-dependent diabetes induced with checkpoint inhibitors. Diabetes 67, 1471-1480 (2018).

77. Akturk,H.K. \& Michels,A.W. Adverse events associated with immune checkpoint blockade. N. Eng. J. Med. 378, 1163-1165 (2018). 
78. Foster,D.W. \& McGarry,J.D. The metabolic derangements and treatment of diabetic ketoacidosis. N. Eng. J. Med. 309, 159-169 (1983).

79. Miles,J.M., Rizza,R.A., Haymond,M.W. \& Gerich,J.E. Effects of acute insulin deficiency on glucose and ketone body turnover in man: evidence for the primacy of overproduction of glucose and ketone bodies in the genesis of diabetic ketoacidosis. Diabetes 29, 926-930 (1980).

80. Gerich,J.E., Meyer,C., Woerle,H.J. \& Stumvoll,M. Renal gluconeogenesis. Diabetes Care 24, 382-391 (2001).

81. Exton,J.H. Gluconeogenesis. Metabolism - Clinical and Experimental 21, 945-990 (1972).

82. Felig,P., Marliss,E., Ohman,J.L. \& Cahill,G.F. Plasma amino acid levels in diabetic ketoacidosis. Diabetes 19, 727-728 (1970).

83. Hatting,M., Tavares,C.D., Sharabi,K., Rines,A.K. \& Puigserver,P. Insulin regulation of gluconeogenesis. Ann. NY. Acad. Sci. 1411, 21-35 (2018).

84. McGarry,J.D., Woeltje,K.F., Kuwajima,M. \& Foster,D.W. Regulation of ketogenesis and the renaissance of carnitine palmitoyltransferase. Diabetes Metab. Rev. 5, 271284 (1989).

85. Foster,D.W. Malonyl-CoA: the regulator of fatty acid synthesis and oxidation. J. Clin. Invest. 122, 1958-1959 (2012).

86. Cook,G.A., King,M.T. \& Veech,R.L. Ketogenesis and malonyl coenzyme A content of isolated rat hepatocytes. J. Biol. Chem. 253, 2529-2531 (1978).

87. Laffel,L. Ketone bodies: a review of physiology, pathophysiology and application of monitoring to diabetes. Diabetes Metab. Res. Rev. 15, 412-426 (1999).

88. Dhatariya,K. Blood ketones - measurement, interpretation, limitations and utility in the management of diabetic ketoacidosis. Rev. Diabet. Stud. 13, 217-225 (2016).

89. Balasse,E.O. \& Fery,F. Ketone body production and disposal: Effects of fasting, diabetes, and exercise. Diabetes Metab. Rev. 5, 247-270 (1989).

90. Kraut,J.A. \& Madias,N.E. Serum anion gap: Its uses and limitations in clinical medicine. Clin. J. Am. Soc. Nephrol. 2, 162-174 (2007).

91. Witte,D.L., Rodgers,J.L. \& Barrett,D.A. The anion gap: its use in quality control. Clin. Chem. 22, 643-646 (1976).

92. Emmett,M. Anion-gap interpretation: the old and the new. Nat. Clin. Pract. Nephrol. 2, 4-5 (2006). 
93. Kamel,K.S. \& Halperin,M.L. Acid-base problems in diabetic ketoacidosis. N. Eng. J. Med. 372, 546-554 (2015).

94. Palmer,B.F. \& Clegg,D.J. Electrolyte and acid-base disturbances in patients with diabetes mellitus. N. Eng. J. Med. 373, 548-559 (2015).

95. Burnell,J.M., Villamil,M.F., Uyeno,B.T. \& Scribner,B.H. The effect in humans of extracellular $\mathrm{pH}$ change on the relationship between sreum potassium and intracellular potassium. J. Clin. Invest. 35, 935-939 (1956).

96. Rains,J.L. \& Jain,S.K. Oxidative stress, insulin signaling, and diabetes. Free Radic. Biol. Med. 50, 567-575 (2011).

97. Li,J., Huang,M. \& Shen,X. The association of oxidative stress and pro-inflammatory cytokines in diabetic patients with hyperglycemic crisis. J. Diabetes Complications 28, 662-666 (2014).

98. Shen,T. \& Braude,S. Changes in serum phosphate during treatment of diabetic ketoacidosis: predictive significance of severity of acidosis on presentation. Intern. Med. J. 42, 1347-1350 (2012).

99. Chaudhuri,A. \& Umpierrez,G.E. Oxidative stress and inflammation in hyperglycemic crises and resolution with insulin: implications for the acute and chronic complications of hyperglycemia. J. Diabetes Complications 26, 257-258 (2012).

100. Roden,M. \& Shulman,G.I. The integrative biology of type 2 diabetes. Nature 576, 51-60 (2019).

101. Saltiel,A.R. \& Olefsky,J.M. Inflammatory mechanisms linking obesity and metabolic disease. J. Clin. Invest. 127, 1-4 (2017).

102. Guilherme,A., Henriques,F., Bedard,A.H. \& Czech,M.P. Molecular pathways linking adipose innervation to insulin action in obesity and diabetes mellitus. Nat. Rev. Endocrinol. 15, 207-225 (2019).

103. Vaarala,O. \& Yki-Jarvinen,H. Should we treat infection or inflammation to prevent T2DM? Nat. Rev. Endocrinol. 8, 323-325 (2012).

104. Pickup,J.C. Inflammation and activated innate immunity in the pathogenesis of type 2 diabetes. Diabetes Care 27, 813-823 (2004).

105. Kim,F. et al. Free fatty acid impairment of nitric oxide production in endothelial cells is mediated by IKKb. Arterioscler Thromb Vasc Biol 25, 989-994 (2005).

106. Stentz,F.B., Umpierrez,G.E., Cuervo,R. \& Kitabchi,A.E. Proinflammatory cytokines, markers of cardiovascular risks, oxidative stress, and lipid peroxidation in patients with hyperglycemic crises. Diabetes 53, 2079-2086 (2004). 
107. Hoffman,W.H., Stamatovic,S.M. \& Andjelkovic,A.V. Inflammatory mediators and blood brain barrier disruption in fatal brain edema of diabetic ketoacidosis. Brain Res. 1254, 138-148 (2009).

108. Glaser,N. et al. Treatment with the KCa3.1 inhibitor TRAM-34 during diabetic ketoacidosis reduces inflammatory changes in the brain. Pediatr. Diabetes 18, 356366 (2017).

109. Omatsu,T. et al. CXCL1/CXCL8 (GROalL-8) in human diabetic ketoacidosis plasma facilitates leukocyte recruitment to cerebrovascular endothelium in vitro. Am. J. Physiol. Endocrinol. Metab. 306, E1077-E1084 (2014).

110. Ferrannini,E., Mark,M. \& Mayoux,E. CV protection in the EMPA-REG OUTCOME Trial: A "thrifty substrate" hypothesis. Diabetes Care 39, 1108-1114 (2016).

111. Ferrannini,E. et al. Shift to fatty substrate utilization in response to Sodium-Glucose Cotransporter 2 inhibition in subjects without diabetes and patients with type 2 diabetes. Diabetes 65, 1190-1195 (2016).

112. Ferrannini,E. Sodium-glucose co-transporters and their inhibition: Clinical physiology. Cell Metabolism 26, 27-38 (2017).

113. Wanner,C. \& Marx,N. SGLT2 inhibitors: the future for treatment of type 2 diabetes mellitus and other chronic diseases. Diabetologia 61, 2134-2139 (2018).

114. Peters,A.L. et al. Euglycemic diabetic ketoacidosis: A potential complication of treatment with sodium-glucose cotransporter 2 inhibition. Diabetes Care 38, 16871693 (2015).

115. Taylor,S.I., Blau,J.E. \& Rother,K.I. Perspective: SGLT2 inhibitors may predispose to ketoacidosis. J Clin Endocrinol Metab 100, 2849-2852 (2015).

116. Palmer,B.F. \& Clegg,D.J. Electrolyte disturbances in patients with chronic alcoholuse disorder. N. Eng. J. Med. 377, 1368-1377 (2017).

117. Umpierrez,G.E. et al. Differences in metabolic and hormonal milieu in diabetic- and alcohol-induced ketoacidosis. J. Crit. Care 15, 52-59 (2000).

118. Reddi,A.S. Acid-Base disorders. Clinical evaluation and management. Reddi,A.S. (ed.), pp. 85-102 (Springer,2019).

119. McGuire,L.C., Cruickshank,A.M. \& Munro,P.T. Alcoholic ketoacidosis. Emerg. Med. J. 23, 417-420 (2006).

120. Cahill,G.F. Fuel metabolism in starvation. Annu. Rev. Nutr. 26, 1-22 (2006).

121. Cahill,G.F. Starvation in man. N. Eng. J. Med. 282, 668-675 (1970). 
122. Owen,O.E. Ketone bodies as a fuel for the brain during starvation. Biochemistry and Molecular Biology Education 33, 246-251 (2005).

123. Wildenhoff,K.E., Ladefoged,K. \& Sorensen,N.S. Clinical physiology: The concentration of ketone bodies, free fatty acids, and glycerol in the blood of obese persons after injection of insulin and glucose studies before and during absolute fasting. Scand. J. Clin. Lab. Invest. 35, 129-133 (1975).

124. Kamel,K.S., Lin,S.H., Cheema-Dhadli,S., Marliss,E.B. \& Halperin,M.L. Prolonged total fasting: A feast for the integrative physiologist. Kidney Int. 53, 531-539 (1998).

125. Xin,Y., Yang,M., Chen,X.J., Tong,Y.J. \& Zhang,L.H. Clinical features at the onset of childhood type 1 diabetes mellitus in Shenyang, China. J. Paediatr. Child Health 46, 171-175 (2010).

126. Umpierrez,G. \& Freire,A.X. Abdominal pain in patients with hyperglycemic crises. J. Crit. Care 17, 63-67 (2002).

127. Umpierrez,G.E., Kelly,J.P., Navarrete,J.E., Casals,M.M. \& Kitabchi,A.E. Hyperglycemic crises in urban blacks. Arch. Intern. Med. 157, 669-675 (1997).

128. Deeter,K.H. et al. Hypertension despite dehydration during severe pediatric diabetic ketoacidosis. Pediatr. Diabetes 12, 295-301 (2011).

129. Dhatariya,K.K. Defining and characterising diabetic ketoacidosis in adults. Diabetes Res Clin Pract 155, 107797 (2019).

130. Savage,M.W. et al. Joint British Diabetes Societies guideline for the management of diabetic ketoacidosis. Diabetic Med. 28, 508-515 (2011).

131. Macfarlane,J. \& Dhatariya,K. The incidence of euglycemic diabetic ketoacidosis in adults with type 1 diabetes in the UK prior to the widespread use of sodium glucose co-transporter 2 inhibitors. Mayo Clin. Proc. 94, 1909-1910 (2019).

132. Munro,J.F., Campbell,I.W., McCuish,A.C. \& Duncan,J.P. Euglycaemic diabetic ketoacidosis. Br. Med. J. 2, 578-580 (1973).

133. Modi,A., Agrawal,A. \& Morgan,F. Euglycemic diabetic ketoacidosis: A review. Curr. Diabetes Rev. 13, 315-321 (2017).

134. Rosenstock,J. \& Ferrannini,E. Euglycemic diabetic ketoacidosis: A predictable, detectable, and preventable safety concern with SGLT2 inhibitors. Diabetes Care 38, 1638-1642 (2015).

135. Adrogue,H.J., Wilson,H., Boyd,A.E., Suki,W.N. \& Eknoyan,G. Plasma acid-base patterns in diabetic ketoacidosis. N. Eng. J. Med. 307, 1603-1610 (1982). 
136. Skellett,S., Mayer,A., Durward,A., Tibby,S.M. \& Murdoch,I.A. Chasing the base deficit: hyperchloraemic acidosis following $0.9 \%$ saline fluid resuscitation. Arch. Dis. Child. 83, 514-516 (2000).

137. Klocker,A.A., Phelan,H., Twigg,S.M. \& Craig,M.E. Blood B-hydroxybutyrate vs. urine acetoacetate testing for the prevention and management of ketoacidosis in Type 1 diabetes: a systematic review. Diabetic Med. 30, 818-824 (2013).

138. Wolfsdorf,J. et al. ISPAD Clinical Practice Consensus Guidelines 2009. Diabetic ketoacidosis. Pediatr. Diabetes 10, 118-133 (2009).

139. Sheikh-Ali,M. et al. Can serum beta-hydroxybutyrate be used to diagnose diabetic ketoacidosis? Diabetes Care 31, 643-647 (2008).

140. Stephens,J.M., Sulway,M.J. \& Watkins,P.J. Relationship of blood acetoacetate and 3-hydroxybutyrate in diabetes. Diabetes 20, 485-489 (1971).

141. Pasquel,F.J. et al. Clinical outcomes in patients with isolated or combined diabetic ketoacidosis and hyperosmolar hyperglycemic state: A retrospective, hospitalbased cohort study. Diabetes Care 43, 349-357 (2020).

142. Teasdale,G. \& Jennett,B. Assessment of coma and impaired consciousness: A practical scale. Lancet 304, 81-84 (1974).

143. Laffel,L. Sick-day management in type 1 diabetes. Endocrinol. Metab. Clin. North Am. 29, 707-723 (2000).

144. Evans,N.R., Richardson,L., Dhatariya,K.K. \& Sampson,M.J. Diabetes specialist nurse telemedicine: admissions avoidance, costs and casemix. Eur. Diabetes Nursing 9, 17-21 (2012).

145. Beran,D., Mirza,Z. \& Dong,J. Access to insulin: applying the concept of security of supply to medicines. Bull. World Health Organ. 97, 309-376 (2019).

146. McLarty,D.G., Kinabo,L. \& Swai,A.B. Diabetes in tropical Africa: a prospective study, 1981-7. II. Course and prognosis. Br. Med. J. 300, 1107-1110 (1990).

147. Shen,X.P., Li,J., Zou,S., Wu,H.J. \& Zhang,Y. The relationship between oxidative stress and the levels of serum circulating adhesion molecules in patients with hyperglycemia crises. J. Diabetes Complications 26, 291-295 (2012).

148. American College of Surgeons Committee on Trauma. Advanced life support course for physicians. American College of Surgeons, Chicago (1993).

149. Karslioglu French,E., Donihi,A.C. \& Korytkowski,M.T. Diabetic ketoacidosis and hyperosmolar hyperglycemic syndrome: review of acute decompensated diabetes in adult patients. BMJ 365, I1114 (2019). 
150. WaldhausI,W. et al. Severe hyperglycemia: Effects of rehydration on endocrine derangements and blood glucose concentration. Diabetes 28, 577-584 (1979).

151. Dhatariya,K.K. Diabetic ketoacidosis. Br. Med. J. 334, 1284-1285 (2007).

152. Van Zyl,D.G., Rheeder,P. \& Delport,E. Fluid management in diabetic-acidosis Ringer's lactate versus normal saline: a randomized controlled trial. QJM 105, 337343 (2012).

153. Kamel,K.S., Schreiber,M., Carlotti,A.P. \& Halperin,M.L. Approach to the treatment of diabetic ketoacidosis. Am. J. Kidney Disease 68, 967-972 (2016).

154. Koves,I.H. et al. The accuracy of clinical assessment of dehydration during diabetic ketoacidosis in childhood. Diabetes Care 27, 2485-2487 (2004).

155. Sottosanti,M. et al. Dehydration in children with diabetic ketoacidosis: a prospective study. Arch. Dis. Child. 97, 96-100 (2012).

156. Ugale,J., Mata,A., Meert,K.L. \& Sarnaik,A.P. Measured degree of dehydration in children and adolescents with type 1 diabetic ketoacidosis. Pediatr. Crit. Care Med. 13, e103-e107 (2012).

157. Duck,S.C. \& Wyatt,D.T. Factors associated with brain herniation in the treatment of diabetic ketoacidosis. J. Pediatr 113, 10-14 (1988).

158. Harris,G.D., Flordalisi,I., Harris,W.L., Mosovich,L.L. \& Finberg,L. Minimizing the risk of brain herniation during treatment of diabetic ketoacidemia: A retrospective and prospective study. J. Pediatr 117, 22-31 (1990).

159. Kuppermann,N. et al. Clinical trial of fluid infusion rates for pediatric diabetic ketoacidosis. N. Eng. J. Med. 378, 2275-2287 (2018).

160. Grimberg,A., Cerri,R.W., Satin-Smith,M. \& Cohen,P. The "two bag system" for variable intravenous dextrose and fluid administration: Benefits in diabetic ketoacidosis management. J. Pediatr 134, 376-378 (1999).

161. Poirier,M.P., Greer,D. \& Satin-Smith,M. A prospective study of the "two-bag system" in diabetic ketoacidosis management. Clin Pediatr (Phila) 43, 809-813 (2004).

162. So,T.Y. \& Grunewalder,E. Evaluation of the two-bag system for fluid management in pediatric patients with diabetic ketoacidosis. J. Pediatr. Pharmacol. Ther. 14, 100105 (2009).

163. Oh,G., Anderson,S., Tancredi,D., Kuppermann,N. \& Glaser,N. Hyponatremia in pediatric diabetic ketoacidosis: Reevaluating the correction factor for hyperglycemia. Arch. Pediatr. Adolesc. Med. 163, 771-772 (2009). 
164. Roscoe,J.M., Halperin,M.L., Rolleston,F.S. \& Goldstein,M.B. Hyperglycemiainduced hyponatremia: metabolic considerations in calculation of serum sodium depression. CMAJ 112, 452-453 (1975).

165. Hillier,T.A., Abbott,R.D. \& Barrett,E.J. Hyponatremia: evaluating the correction factor for hyperglycemia. Am. J. Med. 106, 399-403 (1999).

166. Lindsay,R. \& Bolte,R.G. The use of an insulin bolus in low-dose insulin infusion for pediatric diabetic ketoacidosis. Pediatr. Emerg. Care 5, 77-79 (1989).

167. Kitabchi,A.E., Murphy,M.B., Spencer,J., Matteri,R. \& Karas,J. Is a priming dose of insulin necessary in a low-dose insulin protocol for the treatment of diabetic ketoacidosis? Diabetes Care 31, 2081-2085 (2008).

168. Nallasamy,K., Jayashree,M., Singhi,S. \& Bansal,A. Low-dose vs standard-dose insulin in pediatric diabetic ketoacidosis: A randomized clinical trial. JAMA Pediatrics 168, 999-1005 (2014).

169. Puttha, R. et al. Low dose $(0.05 \mathrm{units} / \mathrm{kg} / \mathrm{h})$ is comparable with standard dose $(0.1$ units $/ \mathrm{kg} / \mathrm{h}$ ) intravenous insulin infusion for the initial treatment of diabetic ketoacidosis in children with type 1 diabetes-an observational study. Pediatr. Diabetes 11, 12-17 (2010).

170. Al Hanshi,S. \& Shann,F. Insulin infused at 0.05 versus $0.1 \mathrm{units} / \mathrm{kg} / \mathrm{hr}$ in children admitted to intensive care with diabetic ketoacidosis. Pediatr. Crit. Care Med. 12, 137-140 (2011).

171. Umpierrez,G.E. et al. Insulin analogs versus human insulin in the treatment of patients with diabetic ketoacidosis. Diabetes Care 32, 1164-1169 (2009).

172. Umpierrez,G.E. et al. Efficacy of subcutaneous insulin lispro versus continuous intravenous regular insulin for the treatment of patients with diabetic ketoacidosis. Am. J. Med. 117, 291-296 (2004).

173. Ersoz,H.O. et al. Subcutaneous lispro and intravenous regular insulin treatments are equally effective and safe for the treatment of mild and moderate diabetic ketoacidosis in adult patients. Int. J. Clin. Pract. 60, 429-433 (2006).

174. Karoli,R., Fatima,J., Salman,T., Sandhu,S. \& Shankar,R. Managing diabetic ketoacidosis in non-intensive care unit setting: Role of insulin analogs. Indian $\mathrm{J}$. Pharmacol. 43, 398-104 (2011).

175. Umpierrez,G.E. et al. Treatment of diabetic ketoacidosis with subcutaneous insulin aspart. Diabetes Care 27, 1873-1878 (2004).

176. Danne,T. et al. ISPAD Clinical Practice Consensus Guidelines 2018: Insulin treatment in children and adolescents with diabetes. Pediatr. Diabetes 19, 115-135 (2018). 
177. Pozzilli,P. et al. Continuous subcutaneous insulin infusion in diabetes: patient populations, safety, efficacy, and pharmacoeconomics. Diabetes Metab. Res. Rev. 32, 21-39 (2016).

178. Pala,L., Dicembrini,I. \& Mannucci,E. Continuous subcutaneous insulin infusion vs modern multiple injection regimens in type 1 diabetes: an updated meta-analysis of randomized clinical trials. Acta Diabetol. 56, 973-980 (2019).

179. Blackman,S.M. et al. Insulin pump use in young children in the T1D Exchange clinic registry is associated with lower hemoglobin A1c levels than injection therapy. Pediatr. Diabetes 15, 564-572 (2014).

180. Kitabchi,A.E., Ayyagari,V. \& Guerra,S.M. The efficacy of low-dose versus conventional therapy of insulin for treatment of diabetic ketoacidosis. Ann. Intern. Med. 84, 633-638 (1976).

181. Sacks,H.S., Shahshahani,M., Kitabchi,A.E., Fisher,J.N. \& Young,R.T. Similar responsiveness of diabetic ketoacidosis to low-dose insulin by intramuscular injection and albumin-free infusion. Ann. Intern. Med. 90, 36-42 (1979).

182. Lever,E. \& Jaspan,J.B. Sodium bicarbonate therapy in severe diabetic ketoacidosis. Am. J. Med. 75, 263-268 (1983).

183. Green,S.M. et al. Failure of adjunctive bicarbonate to improve outcome in severe pediatric diabetic ketoacidosis. Ann. Emerg. Med. 31, 41-48 (1998).

184. Latif,K.A., Freire,A.X., Kitabchi,A.E., Umpierrez,G.E. \& Qureshi,N. The use of alkali therapy in severe diabetic ketoacidosis. Diabetes Care 25, 2113-2114 (2002).

185. Gamba,G., Osequera,J., Casterjon,M. \& Gomez-Perez,F.J. Bicarbonate therapy in severe diabetic ketoacidosis. A double blind, randomized, placebo controlled trial. Rev. Invest. Clin. 43, 234-238 (1991).

186. Glaser,N. et al. Risk factors for cerebral edema in children with diabetic ketoacidosis. N. Eng. J. Med. 344, 264-269 (2001).

187. Fraley,D.S. \& Adler,S. Correction of hyperkalemia by bicarbonate despite constant blood pH. Kidney Int. 12, 354-360 (1977).

188. Ditzel,J. \& Lervang,H. Disturbance of inorganic phosphate metabolism in diabetes mellitus: clinical manifestations of phosphorus-depletion syndrome during recovery from diabetic ketoacidosis. Diabetes Metab. Syndr. Obes. 3, 319-324 (2019).

189. Shilo,S., Werner,D. \& Hershko,C. Acute hemolytic anemia caused by severe hypophosphatemia in diabetic ketoacidosis. Acta Haematol. 73, 55-57 (1985).

190. Choi,H.S. et al. Respiratory failure in a diabetic ketoacidosis patient with severe hypophosphatemia. Ann. Pediatr. Endocrinol. Metab. 23, 103-106 (2018). 
191. Kutlu,A.O., Kara,C. \& Cetinkaya,S. Rhabdomyolysis without detectable myoglobulinuria due to severe hypophosphatemia in diabetic ketoacidosis. Pediatr. Emerg. Care 27, 537-538 (2011).

192. Winter,R.J., Harris,C.J., Phillips,L.S. \& Green,O.C. Diabetic ketoacidosis: Induction of hypocalcemia and hypomagnesemia by phosphate therapy. Am. J. Med. 67, 897900 (1979).

193. Fisher,J.N. \& Kitabchi,A.E. A randomized study of phosphate therapy in the treatment of diabetic ketoacidosis. J. Clin. Endocrinol. Metab. 57, 177-180 (1983).

194. Wilson,H.K., Keuer,S.P., Lea,A.S., Boyd,A. \& Eknoyan,G. Phosphate therapy in diabetic ketoacidosis. Arch. Intern. Med. 142, 517-520 (1982).

195. Edge,J.A. et al. The UK case-control study of cerebral oedema complicating diabetic ketoacidosis in children. Diabetologia 49, 2002-2009 (2006).

196. Lawrence,S.E., Cummings,E.A., Gaboury,I. \& Daneman,D. Population-based study of incidence and risk factors for cerebral edema in pediatric diabetic ketoacidosis. J. Pediatr 146, 688-692 (2005).

197. Krane,E.J., Rockoff,M.A., Wallman,J.K. \& Wolfsdorf,J.I. Subclinical brain swelling in children during treatment of diabetic ketoacidosis. N. Eng. J. Med. 312, 1147-1151 (1985).

198. Glaser,N.S. et al. Mechanism of cerebral edema in children with diabetic ketoacidosis. J. Pediatr 145, 164-171 (2004).

199. Cameron,F.J. et al. Neurological consequences of diabetic ketoacidosis at initial presentation of type 1 diabetes in a prospective cohort study of children. Diabetes Care 37, 1554-1562 (2014).

200. Ghetti,S., Lee,J.K., Sims,C.E., DeMaster,D.M. \& Glaser,N.S. Diabetic ketoacidosis and memory dysfunction in children with type 1 diabetes. J. Pediatr 156, 109-114 (2010).

201. Shehata,G. \& Eltayeb,A. Cognitive function and event-related potentials in children with type 1 diabetes mellitus. J Child Neurol 25, 469-474 (2009).

202. Glasgow,A.M. Devastating cerebral edema in diabetic ketoacidosis before therapy. Diabetes Care 14, 77-78 (1991).

203. Muir,A.B., Quisling,R.G., Yang,M.C. \& Rosenbloom,A.L. Cerebral edema in childhood diabetic ketoacidosis: Natural history, radiographic findings, and early identification. Diabetes Care 27, 1541-1546 (2004). 
204. National Institute for Health and Care Excellence. Type 1 diabetes in adults: diagnosis and management (NG17). https://www.nice.org.uk/guidance/ng17. 2016. [Last accessed 20th March 2020]

205. Peasgood,T. et al. The impact of diabetes-related complications on preferencebased measures of health-related quality of life in adults with type I diabetes. Med Decis Making 36, 1020-1033 (2016).

206. Diabetes UK. End of life diabetes care. https://www.diabetes.org.uk/resourcess3/2018-03/EoL Guidance 2018 Final.pdf. 2018. [Last accessed 20th March 2020]

207. Gutierrez,J.A., Bagatell,R., Samson,M.P., Theodorou,A.A. \& Berg,R.A. Femoral central venous catheter-associated deep venous thrombosis in children with diabetic ketoacidosis. Crit. Care Med. 31, 80-83 (2003).

208. Hursh,B.E., Ronsley,R., Islam,N., Mammen,C. \& Panagiotopoulos,C. Acute kidney injury in children with type 1 diabetes hospitalized for diabetic ketoacidosis. JAMA Pediatrics 171, e170020 (2017).

209. Rammaert,B., Lanternier,F., Poiree,S., Kania,R. \& Lortholary,O. Diabetes and mucormycosis: A complex interplay. Diabetes Metab. 38, 193-204 (2012).

210. Ahmed,M., Healy,M.L., Shea,D. \& Crowley,R.K. Epidural pneumatosis associated with spontaneous pneumomediastinum: a rare complication of diabetic ketoacidosis. BMJ Case Reports 2016, bcr2016216295 (2016).

211. Pain,A.R., Pomroy,J. \& Benjamin,A. Hamman's syndrome in diabetic ketoacidosis. Endocrinol. Diabetes Metab. Case Rep. 2017, 17-0135 (2017).

212. Alsaied,T., Goldstein,S.L., Kaddourah,A. \& Poynter,S.E. Thrombocytopeniaassociated multi-organ failure caused by diabetic ketoacidosis. Pediatr. Int. 58, 232234 (2016).

213. Patra,K.P. \& Scott,L.K. Diabetic ketoacidosis preceding thrombocytopenia associated multiple organ failure in a child. JOP 12, 40-43 (2011).

214. Oschatz,E., Mullner,M., Herkner,H. \& Laggner,A.N. Multiple organ failure and prognosis in adult patients with diabetic ketoacidosis. Wien. Klin. Wochenschr. 111, 590-595 (1999).

215. Baszynska-Wilk,M. et al. Peripheral neuropathy as a complication of diabetic ketoacidosis in a child with newly diagnosed diabetes type 1: A case report. J. Clin. Res. Pediatr. Endocrinol. 10, 289-293 (2018).

216. Hoeijmakers,J.G., Faber,C.G., Miedema,C.J., Merkies,I.S. \& Vles,J.S. Small fiber neuropathy in children: Two case reports illustrating the importance of recognition. Pediatrics 138, e20161215 (2016). 
217. Bonfanti,R. et al. Disseminated intravascular coagulation and severe peripheral neuropathy complicating ketoacidosis in a newly diagnosed diabetic child. Acta Diabetol. 31, 173-174 (1994).

218. Atkin,S.L. et al. Multiple cerebral haematomata and peripheral nerve palsies associated with a case of juvenile diabetic ketoacidosis. Diabetic Med. 12, 267-270 (1995).

219. Mulder,L., Onur,O., Kleis,L., Borders,H. \& Cemeroglu,A.P. Atypical neurologic presentations of new onset type 1 diabetes mellitus in pediatric age group: a report of five unusual cases and review of the literature. J. Ped. Endocrinol. Metab. 27, 749-756 (2014).

220. Fayfman,M., Pasquel,F.J. \& Umpierrez,G.E. Management of hyperglycemic crises: Diabetic ketoacidosis and hyperglycemic hyperosmolar state. Med. Clin. North Am. 101, 587-606 (2017).

221. Elding Larsson, H. et al. Reduced prevalence of diabetic ketoacidosis at diagnosis of type 1 diabetes in young children participating in longitudinal follow-up. Diabetes Care 34, 2347-2352 (2011).

222. Barker,J.M. et al. Clinical characteristics of children diagnosed with type 1 diabetes through intensive screening and follow-up. Diabetes Care 27, 1399-1404 (2004).

223. Vanelli,M. et al. Effectiveness of a prevention program for diabetic ketoacidosis in children. An 8-year study in schools and private practices. Diabetes Care 22, 7-9 (1999).

224. Wagner,D.V., Barry,S.A., Stoeckel,M., Teplitsky,L. \& Harris,M.A. NICH at its best for diabetes at its worst: Texting teens and their caregivers for better outcomes. J. Diabetes Sci. Technol. 11, 468-475 (2017).

225. Wong,J.C. et al. Real-time continuous glucose monitoring among participants in the T1D Exchange Clinic registry. Diabetes Care 37, 2702-2709 (2014).

226. Charleer,S. et al. Effect of continuous glucose monitoring on glycemic control, acute admissions, and quality of life: A real-world study. J. Clin. Endocrinol. Metab. 103, 1224-1232 (2018).

227. Parkin,C.G., Graham,C. \& Smolskis,J. Continuous glucose monitoring use in type 1 diabetes: Longitudinal analysis demonstrates meaningful improvements in $\mathrm{HbA1c}$ and reductions in health care utilization. J. Diabetes Sci. Technol. 11, 522-528 (2017).

228. Norgaard,K. A nationwide study of continuous subcutaneous insulin infusion (CSII) in Denmark. Diabetic Med. 20, 307-311 (2003). 
229. Dogan,A.D., Jorgensen,U.L. \& Gjessing,H.J. Diabetic ketoacidosis among patients treated with continuous subcutaneous insulin infusion. J. Diabetes Sci. Technol. 11, 631-632 (2017).

230. Cengiz,E. et al. Severe hypoglycemia and diabetic ketoacidosis among youth with type 1 diabetes in the T1D Exchange clinic registry. Pediatr. Diabetes 14, 447-454 (2013).

231. Karges,B., Schwandt,A. \& Heidtmann,B. Association of insulin pump therapy vs insulin injection therapy with severe hypoglycemia, ketoacidosis, and glycemic control among children, adolescents, and young adults with type 1 diabetes. JAMA 318, 1358-1366 (2017).

232. Elliot,J. et al. Substantial reductions in the number of diabetic ketoacidosis and severe hypoglycaemia episodes requiring emergency treatment lead to reduced costs after structured education in adults with Type 1 diabetes. Diabetic Med. 31, 847-853 (2014).

233. Ilkowitz,J.T., Choi,S., Rinke,M.L., Vandervoot,K. \& Heptulla,R.A. Pediatric type 1 diabetes: Reducing admission rates for diabetes ketoacidosis. Qual. Manag. Health Care 25, 231-237 (2016).

234. World Health Organization. Essential medicines and health products information portal. WHO model formulary, 2008. Based on the 15th model list of essential medicines 2007. http://apps.who.int/medicinedocs/en/m/abstract/Js16879e/. 2009. [Last accessed 20th March 2020]

235. Williams,V., Jayashree,M., Nallasamy,K., Dayal,D. \& Rawat,A. 0.9\% saline versus Plasma-Lyte as initial fluid in children with diabetic ketoacidosis (SPinK trial): a double-blind randomized controlled trial. Critical Care (London) 24, 1 (2020).

236. Hsia,E. et al. Subcutaneous administration of glargine to diabetic patients receiving insulin infusion prevents rebound hyperglycemia. J. Clin. Endocrinol. Metab. 97, 3132-3137 (2012).

237. Bekiari,E. et al. Artificial pancreas treatment for outpatients with type 1 diabetes: systematic review and meta-analysis. BMJ 361, k1310 (2018).

238. Karageorgiou, $\mathrm{V}$. et al. Effectiveness of artificial pancreas in the non-adult population: A systematic review and network meta-analysis. Metabolism - Clinical and Experimental 90, 20-30 (2019).

239. Meaden,C.W., Kushner,B.J. \& Barnes,S. A rare and lethal complication: Cerebral edema in the adult patient with diabetic ketoacidosis. Case Rep. Emerg. Med. 5043752, https://doi.org/10.1155/2018/5043752 (2019).

240. Nao,J., Zhang,H., Wu,S., Zhang,X. \& Zheng,D. Posterior reversible encephalopathy syndrome with spinal cord involvement (PRES-SCI) as a rare complication of 
severe diabetic ketoacidosis: a case report and review of the literature. Childs. Nerv. Syst. 34, 701-705 (2018).

241. Finn,B.P. et al. Subarachnoid and parenchymal haemorrhages as a complication of severe diabetic ketoacidosis in a preadolescent with new onset type 1 diabetes. Pediatr. Diabetes 19, 1487-1491 (2018).

242. Weissbach,A. et al. Acute kidney injury in critically ill children admitted to the PICU for diabetic ketoacidosis. A retrospective study. Pediatr. Crit. Care Med. 20, e10e14 (2019).

243. Orban,J.C., Maiziere,E.M., Ghaddab,A., Van Obberghen,E. \& Ichai,C. Incidence and characteristics of acute kidney injury in severe diabetic ketoacidosis. PLOS ONE 9, e110925 (2014).

244. Scordi-Bello,I., Kirsch,D. \& Hammers,J. Fatal pulmonary thromboembolism in patients with diabetic ketoacidosis: A seven-case series and review of the literature. Acad. Forensic Pathol. 6, 198-205 (2016).

245. Wakabayashi,S. et al. Acute multiple arteriovenous thromboses in a patient with diabetic ketoacidosis. Intern. Med. 54, 2025-2028 (2015).

246. Jorgensen,L.B., Skov,O. \& Yderstraede,K. Newly diagnosed type 1 diabetes complicated by ketoacidosis and peripheral thrombosis leading to transfemoral amputation. BMJ Case Reports 2014, bcr2013202139 (2014).

247. Cherian,S.V. et al. Diabetic ketoacidosis complicated by generalized venous thrombosis: a case report and review. Blood Coagul. Fibrinolysis 23, 238-240 (2012).

248. Dixon,A.N., Jude,E.B., Banerjee,A.K. \& Bain,S.C. Simultaneous pulmonary and cerebral oedema, and multiple CNS infarctions as complications of diabetic ketoacidosis: a case report. Diabetic Med. 23, 571-573 (2006).

249. Young,M.C. Simultaneous acute cerebral and pulmonary edema complicating diabetic ketoacidosis. Diabetes Care 18, 1288-1290 (1995).

250. Quiros,J. et al. Elevated serum amylase and lipase in pediatric diabetic ketoacidosis. Pediatr. Crit. Care Med. 9, 418-422 (2008).

251. Nair,S., Yadav,D. \& Pitchumoni,C. Association of diabetic ketoacidosis and acute pancreatitis: Observations in 100 consecutive episodes of DKA. Am. J. Gastroenterol. 95, 2795-2800 (2000).

252. Yadav,D., Nair,S., Norkus,E. \& Pitchumoni,C. Nonspecific hyperamylasemia and hyperlipasemia in diabetic ketoacidosis: Incidence and correlation with biochemical abnormalities. Am. J. Gastroenterol. 95, 3123-3128 (2000). 
253. Finn,B.P., Fraser,B. \& O'Connell,S.M. Supraventricular tachycardia as a complication of severe diabetic ketoacidosis in an adolescent with new-onset type 1 diabetes. BMJ Case Reports 2018, bcr-2017 (2018).

254. Miszczuk,K. et al. Ventricular bigeminy and trigeminy caused by hypophosphataemia during diabetic ketoacidosis treatment: a case report. Ital. J. Pediatr. 45, 42 (2019).

255. McGreevy,M., Beerman,L. \& Arora,G. Ventricular tachycardia in a child with diabetic ketoacidosis without heart disease. Cardiol. Young 26, 206-208 (2016).

256. Abdulaziz,S., Dabbagh,O., Al Daker,M.O. \& Hassan,I. Hypokalaemia and refractory asystole complicating diabetic ketoacidosis, lessons for prevention. BMJ Case Reports 2012, bcr-2012 (2012).

257. Alanzalon,R.E., Burris,J.R. \& Vinocur,J.M. Brugada phenocopy associated with diabetic ketoacidosis in two pediatric patients. J. Electrocardiol. 51, 323-326 (2018).

258. Haseeb,S. et al. Brugada pattern in diabetic ketoacidosis: A case report and scoping study. Am. J. Med. Case Rep. 6, 173-179 (2018).

259. Hoffman,W.H. et al. Increased systemic Th17 cytokines are associated with diastolic dysfunction in children and adolescents with diabetic ketoacidosis. PLOS ONE 8, e71905 (2013).

260. Atabek,M.E., Pirgon,O., Oran,B., Erkul,I. \& Kurtoglu,S. Increased cardiac troponin I concentration in diabetic ketoacidosis. J. Ped. Endocrinol. Metab. 17, 1077-1082 (2004).

261. Halloum,A. \& Al Neyadi,S. Myocardial dysfunction associated with diabetic ketoacidosis in a 5-year-old girl. SAGE Open Med. Case Rep. 7, 2050313X19847797 (2019).

262. Odubanjo,A.A. et al. Severe myopericarditis in diabetic ketoacidosis - all troponin are not myocardial infarction. Clin Med Insights Case Rep 11, 1179547618763356 (2018).

263. Casteels,K., Beckers,D., Wouters,C. \& Van Geet,C. Rhabdomyolysis in diabetic ketoacidosis. Pediatr. Diabetes 4, 29-31 (2003).

264. Higa,E.M., Dib,S.A., Martins,J.R., Campos,L. \& Homsi,E. Acute renal failure due to rhabdomyolysis in diabetic patients. Renal Failure 19, 289-293 (1997).

265. Buckingham,B.A., Roe,T.F. \& Yoon,J.W. Rhabdomyolysis in diabetic ketoacidosis. JAMA Pediatrics 135, 352-354 (1981).

266. Wang,L.M., Tsai,S.T., Ho,L.T., Hu,S.C. \& Lee,C.H. Rhabdomyolysis in diabetic emergencies. Diabetes Res Clin Pract 26, 209-214 (1994). 
267. DiMeglio,L.A., Chaet,M.S., Quigley,C.A. \& Grosfeld,J.L. Massive ischemic intestinal necrosis at the onset of diabetes mellitus with ketoacidosis in a three-year-old girl. J. Ped. Surg. 38, 1537-1539 (2003).

268. Chan-Cua,S., Jones,K.L., Lynch,F.P. \& Freidenberg,G.R. Necrosis of the ileum in a diabetic adolescent. J. Ped. Surg. 27, 1236-1238 (1992).

269. Pasquel,F.J. \& Umpierrez,G.E. Hyperosmolar hyperglycemic state: A historic review of the clinical presentation, diagnosis, and treatment. Diabetes Care 37, 3124-3131 (2014).

270. Munson,E.L. The chemistry of the urine in diabetes mellitus. JAMA 28, 831-836 (1897).

271. Kussmaul,A., Foulis,D. \& Gemmell,S. On a peculiar mode of death in diabetes; on acetonæmia; on the treatment of diabetes by glycerine, and injection of diastase into the blood. GMJ 6, 485-500 (1874).

272. Stadelmann,E. Ueber die ursachen der pathologischen ammoniakausscheidung beim diabetes mellitus und des coma diabeticum. Archiv fur experimentelle Pathologie und Pharmakologie 17, 419-444 (1883).

273. Butler,A.M. Diabetic coma. N. Eng. J. Med. 243, 648-659 (1950).

274. Page,M.M. et al. Treatment of diabetic coma with continuous low-dose infusion of insulin. Br. Med. J. 2, 687-690 (1974).

275. Scott,A. \& on Behalf of the Joint British Diabetes Societies (JBDS) for Inpatient Care. Management of hyperosmolar hyperglycaemic state in adults with diabetes. Diabetic Med. 32, 714-724 (2015).

276. Roberts,A., James,J., Dhatariya,K. \& behalf of the Joint British Diabetes Societies (JBDS) for Inpatient Care group and guidelines writing group. Management of hyperglycaemia and steroid (glucocorticoid) therapy: a guideline from the Joint British Diabetes Societies (JBDS) for Inpatient Care group. Diabetic Med. 35, 10111017 (2018).

277. Holt,R.I. Association between antipsychotic medication use and diabetes. Curr. Diab. Rep. 19, 96 (2019).

278. Pasquel,F.J. et al. Clinical outcomes in patients with isolated or combined diabetic ketoacidosis and hyperosmolar hyperglycemic state: A retrospective, hospitalbased cohort study. Diabetes Care. 43, 349-357 (2020). 